\title{
Holographic Floquet states I: a strongly coupled Weyl semimetal
}

\author{
Koji Hashimoto, ${ }^{a}$ Shunichiro Kinoshita, ${ }^{b}$ Keiju Murata ${ }^{c}$ and Takashi Oka ${ }^{d, e}$ \\ ${ }^{a}$ Department of Physics, Osaka University, Toyonaka, Osaka 560-0043, Japan \\ ${ }^{b}$ Department of Physics, Chuo University, Tokyo 112-8551, Japan \\ ${ }^{c}$ Keio University, 4-1-1 Hiyoshi, Yokohama 223-8521, Japan \\ ${ }^{d}$ Max-Planck-Institut für Physik komplexer Systeme (MPI-PKS), \\ Nöthnitzer Straße 38, Dresden 01187, Germany \\ ${ }^{e}$ Max-Planck-Institut für Chemische Physik fester Stoffe (MPI-CPfS), \\ Nöthnitzer Straße 40, Dresden 0118\%, Germany \\ E-mail: koji@phys.sci.osaka-u.ac.jp, kinoshita@phys.chuo-u.ac.jp, \\ keiju@phys-h.keio.ac.jp, oka@pks.mpg.de
}

ABSTRACT: Floquet states can be realized in quantum systems driven by continuous timeperiodic perturbations. It is known that a state known as the Floquet Weyl semimetal can be realized when free Dirac fermions are placed in a rotating electric field. What will happen if strong interaction is introduced to this system? Will the interaction wash out the characteristic features of Weyl semimetals such as the Hall response? Is there a steady state and what is its thermodynamic behavior? We answer these questions using AdS/CFT correspondence in the $\mathcal{N}=2$ supersymmetric massless QCD in a rotating electric field in the large $N_{c}$ limit realizing the first example of a "holographic Floquet state". In this limit, gluons not only mediate interaction, but also act as an energy reservoir and stabilize the nonequilibrium steady state (NESS). We obtain the electric current induced by a rotating electric field: in the high frequency region, the Ohm's law is satisfied, while we recover the DC nonlinear conductivity at low frequency, which was obtained holographically in a previous work. The thermodynamic properties of the NESS, e.g., fluctuation-dissipation relation, is characterized by the effective Hawking temperature that is defined from the effective horizon giving a holographic meaning to the "periodic thermodynamic" concept. In addition to the strong (pump) rotating electric field, we apply an additional weak (probe) electric field in the spirit of the pump-probe experiments done in condensed matter experiments. Weak DC and AC probe analysis in the background rotating electric field shows Hall currents as a linear response, therefore the Hall response of Floquet Weyl semimetals survives at the strong coupling limit. We also find frequency mixed response currents, i.e., a heterodyning effect, characteristic to periodically driven Floquet systems.

KEYwORDS: AdS-CFT Correspondence, Gauge-gravity correspondence, Holography and condensed matter physics (AdS/CMT), Holography and quark-gluon plasmas

ArXIV EPRINT: 1611.03702 


\section{Contents}

1 Introduction 1

2 Floquet Weyl semimetal at weak coupling 4

3 Floquet state in AdS/CFT $\quad 6$

3.1 Set up 6

3.2 Boundary conditions at the effective horizon and the AdS boundary 9

$\begin{array}{lll}3.3 & \text { Effective metric and temperature } & 10\end{array}$

3.4 Physical quantities of the holographic Floquet state 13

4 Hall effect of the holographic Floquet state 14

$\begin{array}{ll}4.1 \text { Conductivities } & 14\end{array}$

$\begin{array}{lll}4.2 & \text { DC Hall effect } & 16\end{array}$

4.3 Optical Hall effect 18

5 Conclusion and discussion $\quad 20$

$\begin{array}{ll}\text { A Regular solution near the effective horizon } & 21\end{array}$

B Perturbation equations $\quad 22$

C Relations in conductivity matrices $\quad 24$

D Numerical method for the time domain approach $\quad 24$

$\begin{array}{ll}\text { E Other conductivities } & 25\end{array}$

\section{Introduction}

Nonequilibrium phenomenon in strongly correlated systems is of general interest and the machinery of the renowned AdS/CFT correspondence [1-3] can assist us reveal its nature (Its application to equilibrium condensed matter problems can be found in refs. [4-6]). In this article, we apply it to study the massless QCD in a rotating electric field

$$
\vec{E}(t)=\left(\begin{array}{c}
\cos \Omega t \\
\sin \Omega t
\end{array}\right) E, \quad(E \geq 0)
$$

where the field with constant strength $E$ is rotating anticlockwise with a frequency $\Omega$ in the $(x, y)$-plane. The schematic picture of the setup is shown in figure 1. This background field drives the system away from equilibrium and generates a nonequilibrium state with broken time reversal symmetry. When a free Dirac particle in $(3+1)$ dimensions with a Lagrangian $\mathcal{L}=\bar{\psi}(i \not \partial-e A) \psi\left(\vec{E}=-\partial_{t} \vec{A}, A=\gamma^{\mu} A_{\mu}\right.$ and $\gamma^{\mu}$ are gamma matrices $)$ is considered, the field will dynamically generate a constant axial vector field in the $z$-direction. The resulting static effective Lagrangian is given by

$$
\mathcal{L}_{\text {eff }}=\bar{\psi} i \not \partial \psi-b \bar{\psi} \gamma_{5} \gamma^{z} \psi
$$




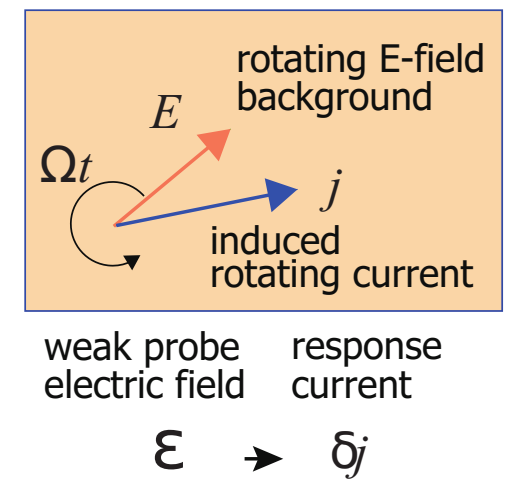

Figure 1. Setup for the holographic calculation. In the steady state, the rotating electric field $E$ within the $(x, y)$-plane induces a retarded rotating current $j$ in the $(x, y)$-plane. In addition to $E$, a weak non-rotating electric field $\varepsilon$ is applied. This field induces a current $\delta j$ linear in $\varepsilon$.

with $b=(e E)^{2} / \Omega^{3}+\mathcal{O}\left(1 / \Omega^{5}\right)$ [7]. Floquet theorem is a temporal analogue of the Bloch theorem and enables us to systematically study periodically driven states by mapping it to a time-independent effective problem with the aid of Fourier mode expansion [8]. Using proper driving fields, it is possible to control the topological properties of the system since we can induce adequate terms necessary to realize non-trivial topology in the spectrum as in the axial vector field $b$ in (1.2). The study of "Floquet topological insulators" [9-12] is becoming a hot topic in condensed matter systems and recently Haldane's topological lattice model [13] was experimentally realized [14] by applying a rotating electric field to fermions in a honeycomb lattice [10]. In $(2+1)$ dimensions, the free massless Dirac particle shows a gap opening in rotating electric fields leading to an emergent parity anomaly (Hall effect), where the direction of the Hall current, i.e., a current flowing perpendicular to the applied DC-electric field, can be controlled by changing the polarization of the field. The gap opening in (2+1)-dimensional Dirac fermions was already experimentally observed in an ultrafast pump-probe experiment [15]. Now, moving on to a (3+1)-dimensional Dirac system, an analogous phenomena take place and it was predicted that the Dirac point splits into two Weyl points [16] forming a "Floquet Weyl semimetal" [17] with broken time reversal symmetry followed by refs. [7, 18-20]. ${ }^{1}$ Figure $2(\mathrm{a}-\mathrm{e})$ shows the numerically exact Floquet quasienergy and we clearly see the two Weyl points in (b) (see section 2 for further details).

At least in the weak coupling limit, Floquet Weyl semimetals can be created from Dirac semimetals by applying the rotating electric field. Here, we arise following questions. What will happen in a strongly interacting fermion systems? Will the interaction wash out the characteristic features of Weyl semimetals such as Hall response? Is there a nonequilibrium steady state and what is the thermodynamic behavior? We address these questions using AdS/CFT correspondence. "Periodic thermodynamics" is another important topic in the research of periodically driven many-body systems [22-25]. Whether or not thermodynamic concepts such as the variational principle, temperature and universal distributions, e.g.,

\footnotetext{
${ }^{1}$ It was also predicted that a circularly polarized light drives nodal line semimetals, characterized by linear band touching at one-dimensional lines, into Weyl semimetals [21].
} 


\section{non-interacting case}

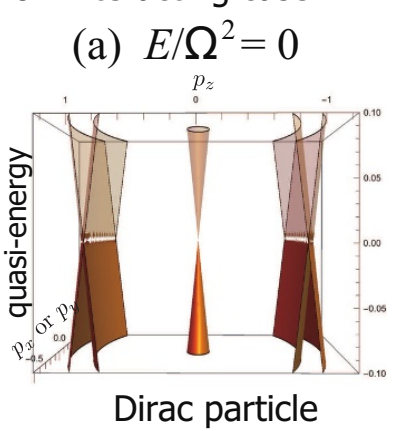

(b) $E / \Omega^{2}=0.25$

(c) $E / \Omega^{2}=0.48$
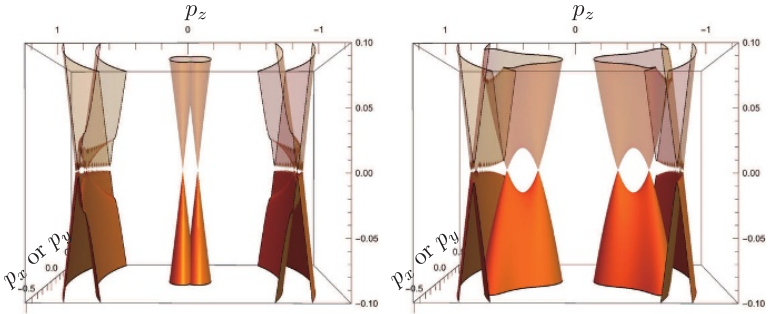

Floquet Weyl semimetal

(d) $E / \Omega^{2}=0.5$

(e) $E / \Omega^{2}=0.53$

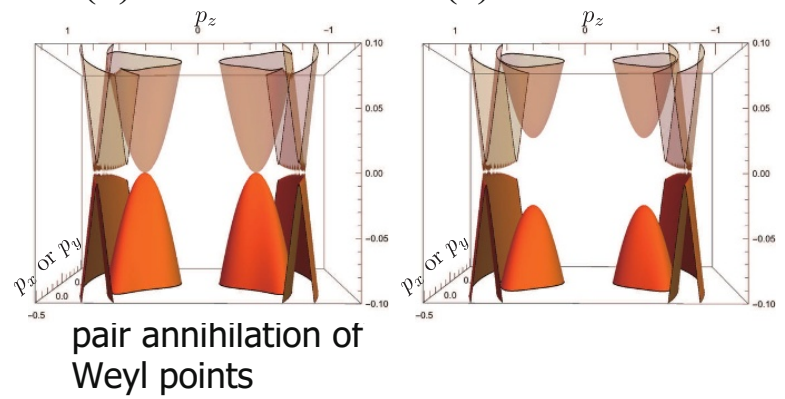

Figure 2. Quasi-energy spectrum of a noninteracting Dirac particle in rotating electric fields, taken from [20]. (a) At zero field, spectrum of massless Dirac particles exists at $\vec{p}=0$ and the Floquet sidebands for states with energies $\pm|\vec{p}|+m \Omega$ surrounds it ( $m$ : integer). (b,c) For fields $0<E / \Omega^{2} \leq 1 / 2$, the Dirac spectrum splits into spectra of two Weyl fermions (1.2). At $p_{z}= \pm 1$, $p_{x}=p_{y}=0$, a new sets of Weyl points emerge and move toward the two Weyl points near the center. (d) The central Weyl points pair annihilates with the emergent Weyl points at fields $E / \Omega^{2}=1 / 2$. (e) A gap opens at $p_{z}= \pm 1 / 2, p_{x}=p_{y}=0$.

Gibbs ensemble, still has a meaning in driven systems is an interesting question. To realize "periodic thermodynamics", it is necessary to stabilize the system because it is constantly heated up by the driving. One way to fulfill this is to attach the system to a heat reservoir [22], while Floquet topological states coupled to a boson bath has been studied in refs. [26, 27].

AdS/CFT correspondence can provide a framework to study these problems in a strongly coupled field theory. As a toy model, we focus on $\mathcal{N}=2 \mathrm{SU}\left(N_{c}\right)$ supersymmetric QCD at large $N_{c}$ and 't Hooft coupling. This theory is geometrically realized as a probe D7-brane in $\mathrm{AdS}_{5} \times S^{5}$ spacetime [28]. The low energy theory has a single quark field (D7-brane) and $\mathrm{SU}\left(N_{c}\right)$ gluon fields $\left(\mathrm{AdS}_{5} \times S^{5}\right.$ background) that mediates interaction. In order to mimic the Floquet Weyl semimetal, we set the quark to be massless and apply the external rotating electric field. ${ }^{2}$ Since there are infinite number of gluon fields in the large $N_{c}$ limit, the gluons not only mediate a long range interaction between the quarks, but also acts as a thermal reservoir. Thus, a nonequilibrium steady state (NESS) may be stabilized even if the system is constantly heated up by external driving.

\footnotetext{
${ }^{2}$ A bottom-up holographic model for the Weyl semimetal was proposed in ref. [29].
} 
We will demonstrate first analysis of the nonlinear response against the strong and rotating electric field applied to $\mathcal{N}=2$ supersymmetric QCD via the AdS/CFT correspondence. Let us briefly look at relevant history of calculations of nonlinear and linear responses against electric field in gravity models to illustrate our analysis. A strong and constant electric field in D3-D7 model was first considered in refs. [30-32]. The quark electric current $j$ was found to be proportional to $E^{3 / 2}$ for the case of massless supersymmetric QCD, as a consequence of a scale invariance of the theory. For the case of massive QCD, the nonlinear regime is indispensable also for a "deconfinement" transition [31-35]. (See also refs. [36-38] for the dynamical phase transition by the electric field quench in the D3-D7 model.) The probe AC conductivities have also been widely studied in AdS/CFT especially in its application to condensed matter physics. For example, in the holographic superconductor [39, 40], AC conductivity under a weak AC electric field was calculated, and a superconductivity gap was explicitly obtained. (See refs. [41-43] for comprehensive reviews on the AC conductivities in various gravity models using AdS/CFT.) The nonlinear response against strong AC electric field in the holographic superconductor is also studied in refs. [44, 45]. For the strong and time-dependent electric field, its response has not been studied so much because of its technical difficulty: we need to solve nonlinear partial differential equations obtained in the gravity side. We overcome the difficulty by considering the rotating electric field. We will see that the resultant equations of motion reduce to ordinary differential equations for the rotating electric field even in the nonlinear regime.

This paper is organized as follows. First, in section 2, we shall introduce a free fermion picture of Weyl semimetal under a rotating electric field, using Floquet method. Then in section 3 , we describe the gravity dual of strongly coupled $\mathcal{N}=2$ supersymmetric massless QCD at large $N_{c}$, and introduce the rotating external electric field. We obtain the nonlinear conductivity explicitly as a function of the intensity of the electric field and the frequency $\Omega$. In particular, in the DC limit $\Omega \rightarrow 0$, our result reproduces the $j \propto E^{3 / 2}$ law found above, while in the other limit $\Omega \rightarrow \infty$ we find that the system is subject to the Ohm's law. In section 4, we calculate linear DC and AC response to the background rotating electric field, and discover a holographic Hall effect: electric currents can have a component perpendicular to the introduced (probe) weak DC and AC electric fields. We also find frequency mixed response currents characteristic to periodically driven Floquet systems. Appendices are given for explicit notations and numerical recipes.

\section{Floquet Weyl semimetal at weak coupling}

Here, we describe the Floquet spectrum of a free massless Dirac particle in a rotating electric field $[7,17]$. In the rotating electric field the Hamiltonian $H(t)$ becomes periodically timedependent, i.e., $H(t+T)=H(t)$ where $T=2 \pi / \Omega$ is the periodicity. Quantum states in time periodic driving are described by the Floquet theory $[8,47]$, that is, a temporal version of the Bloch theorem. The essence of the Floquet theory is a mapping between the time-dependent Schrödinger equation and a static eigenvalue problem. The eigenvalue is called the Floquet pseudo-energy and plays a role similar to the energy in a static system. 
Let us consider a Hamiltonian, $H_{\mathrm{tot}}=H_{0}+H_{\mathrm{bg}}$, with

$$
H_{0}=\gamma^{0} \boldsymbol{\gamma} \cdot \boldsymbol{p}+\gamma^{0} m, \quad H_{\mathrm{bg}}=-e \gamma^{0} \boldsymbol{\gamma} \cdot \boldsymbol{A}
$$

that describes the one-particle Dirac system coupled to an external background gauge field and $\gamma^{\mu}$ are the Dirac matrices satisfying $\left\{\gamma^{\mu}, \gamma^{\nu}\right\}=2 \eta^{\mu \nu}$. In an rotating electric field in the $(x, y)$-plane $(1.1)$, we can write the time-dependent vector potential as

$$
A_{x}=-\frac{E}{\Omega} \sin (\Omega t), \quad A_{y}=\frac{E}{\Omega} \cos (\Omega t), \quad A_{z}=0,
$$

where $\Omega$ is the frequency. We can conveniently decompose the interaction part of the Hamiltonian into two pieces as $H_{\mathrm{bg}}=e^{i \Omega t} H_{-}+e^{-i \Omega t} H_{+}$where $H_{ \pm}= \pm i(e E / \Omega) \gamma^{0} \gamma^{ \pm}$with $\gamma^{ \pm}=\frac{1}{2}\left(\gamma^{x} \pm i \gamma^{y}\right)$. For a periodically time-dependent Hamiltonian $H_{\text {tot }}(t+T)=H_{\text {tot }}(t)$, from the Floquet theorem, a solution of the time-dependent Schrödinger equation $i \partial_{t} \psi_{n}(t)=H_{\text {tot }}(t) \psi_{n}(t)$ can be written as $\psi_{n}(t)=e^{-i \epsilon_{n} t} \Phi_{n}(t)$ where $\Phi_{n}(t+T)=\Phi_{n}(t)$ is the time-periodic Floquet state and $n$ labels the independent solutions. Here, $\epsilon_{n}$ is called the Floquet quasi-energy. Since $\psi_{n}$ satisfies the time-dependent Schrödinger equation, we find that the Floquet state satisfies an eigenvalue problem $\mathcal{H} \Phi_{n}=\epsilon_{n} \Phi_{n}$ where $\mathcal{H}=H_{\text {tot }}-i \partial_{t}$ is a Hermitian operator that acts on the space of time periodic functions: $\Phi(t+T)=\Phi(t)$. Thus, even in a time-dependent system, when the Hamiltonian is time periodic, we can define a quasi-energy spectrum $\epsilon_{n}(\boldsymbol{p})$. Using discrete Fourier basis (see ref. [20] for details) one can efficiently obtain the spectrum as plotted in figure 2.

Now we assume that the period $T=2 \pi / \Omega$ of the circular polarization is small enough as compared to the typical observation timescale. We can then expand the theory in terms of $\omega / \Omega$ (with $\omega$ being a frequency corresponding to some excitation energy). Taking the average over $T$ we can readily find the following effective Hamiltonian by the Floquet Magnus expansion:

$$
\begin{aligned}
H_{\mathrm{eff}} & =\frac{i}{T} \ln \left[\mathcal{T} e^{-i \int_{0}^{T} d t H(t)}\right] \\
& =\frac{1}{T} \int_{0}^{T} d t H(t)+\frac{1}{2 ! T i} \int_{0}^{T} d t_{1} \int_{0}^{t_{1}} d t_{2}\left[H\left(t_{1}\right), H\left(t_{2}\right)\right]+\mathcal{O}\left(T^{-2}\right), \\
& =H_{0}+\frac{1}{\Omega}\left[H_{-}, H_{+}\right]+\mathcal{O}\left(T^{-2}\right),
\end{aligned}
$$

to the first order in the expansion [12]..$^{3}$ Interestingly we can express the induced term as

$$
H_{\text {ind }} \equiv \frac{1}{\Omega}\left[H_{-}, H_{+}\right]=-\frac{(e E)^{2}}{\Omega^{3}} i \gamma^{x} \gamma^{y}=\beta \gamma^{0} \gamma_{5} \gamma^{z},
$$

where we defined $\beta \equiv(e E)^{2} / \Omega^{3}$. This means that the rotating electric field induces an axial-vector background field $\boldsymbol{A}_{5}=\beta \hat{\boldsymbol{z}}$ perpendicular to the polarization plane [7]. An equivalent term as (2.6) was first obtained in ref. [17] in the context of "Floquet Weyl semimetal."

\footnotetext{
${ }^{3}$ In the expansion $(2.3)$, the terms $\left[H_{0}, H_{+}\right]$and $\left[H_{0}, H_{-}\right]$are dropped. These terms, breaking rotational symmetry, can be gauge out through a unitary transformation that corresponds to changing the initial direction of the rotating electric field.
} 
The effect of finite $\beta$ is easily understandable from the energy dispersion relations. We can immediately diagonalize $H_{\text {eff }}$ and the four pseudo-energies read:

$$
\varepsilon_{ \pm}(p)=\sqrt{p_{x}^{2}+p_{y}^{2}+\left(\sqrt{p_{z}^{2}+m^{2}} \pm \beta\right)^{2}}
$$

and $-\varepsilon_{ \pm}(p)$. It shows that the Dirac point splits into two Weyl points with a displacement given by

$$
\Delta p=\sqrt{\beta^{2}-m^{2}} .
$$

In fact, $\beta$ is nothing but a momentum shift along the $z$-axis that is positive for the right chirality state (i.e., $\gamma_{5} \psi_{\mathrm{R}}=+\psi_{\mathrm{R}}$ ) and negative for the left chirality state (i.e., $\gamma_{5} \psi_{\mathrm{L}}=-\psi_{\mathrm{L}}$ ). We point out that time- and angle-resolved photoemission spectroscopy should be able to see this splitting of Weyl points in a similar manner as the gap opening [10] of the $(2+1)$-dimensional Dirac point already observed experimentally [15]. Interestingly, as long as $\beta>m$, the pseudo-energy always has two Weyl points (if they are inside of the Brillouin zone) even for $m>0$. Therefore, we do not have to require strict massless-ness to realize gapless dispersions, which should be a quite useful feature for practical applications including the Schwinger or Landau-Zener effect.

The full Floquet spectrum for $m=0$ is obtained numerically by diagonalizing the Floquet Hamiltonian [20]. The spectrum is given in figures 2. Here we briefly describe and summarize features of the spectrum:

(a) $\boldsymbol{E} / \boldsymbol{\Omega}^{\mathbf{2}}=\mathbf{0}$ : the Dirac point exists at $\vec{p}=0$. The degeneracy of this point is 4 .

(b)(c) $0<\boldsymbol{E} / \boldsymbol{\Omega}^{\mathbf{2}}<\mathbf{0 . 5}$ : four Weyl points exists. Two Weyl points came from the initial Dirac point while the other 2 comes from the hybridized state between the 1 photon absorbed and emitted states.

(d) $\boldsymbol{E} / \boldsymbol{\Omega}^{\mathbf{2}}=\mathbf{0 . 5}$ : the two Weyl points vanish through pair annihilation with the emergent Weyl points originating from the Floquet side bands. ${ }^{4}$ Two parabolic Dirac points appear at $p_{x}=p_{y}=0, p_{z}= \pm 0.5$.

(e) $\boldsymbol{E} / \boldsymbol{\Omega}^{2}>\mathbf{0 . 5}$ : gap opens at the Dirac points.

The full result is consistent with the perturbative result. Applying the rotating electric field shifts the location of the Weyl points, and the Dirac point is separated into two Weyl nodes.

\section{Floquet state in AdS/CFT}

\subsection{Set up}

In the previous section, we have argued that, in the weak coupling limit, the Floquet Weyl semimetal can be created from the Dirac semimetal by a rotating electric field. Here, we consider a similar set up in the strong coupling limit using AdS/CFT correspondence. As a

\footnotetext{
${ }^{4}$ Pair annihilation of Weyl points with opposite chiralities is one way to deform the Weyl system. Another mechanism is interaction: it was proposed that forward scattering can open a gap in the Weyl spectrum [48].
} 
toy model of the strongly coupled field theory, we focus on $\mathcal{N}=2 \mathrm{SU}\left(N_{c}\right)$ supersymmetric QCD at large $N_{c}$ and at strong 't Hooft coupling. Using AdS/CFT correspondence, $\mathcal{N}=2$ SQCD is realized by the probe D7-brane in $\mathrm{AdS}_{5} \times S^{5}$ spacetime [28]. We expect that quarks and antiquarks in this theory play the roles of electrons and holes in condensed matter systems. Applying the rotating external electric field in the system, we will realize the gravity dual of a Floquet state in $\mathcal{N}=2$ SQCD. We use the following coordinates for the $\mathrm{AdS}_{5} \times S^{5}$ spacetime as

$$
\begin{aligned}
d s^{2}= & \frac{\rho^{2}+w_{1}^{2}+w_{2}^{2}}{R^{2}}\left[-d t^{2}+d x^{2}+d y^{2}+d z^{2}\right] \\
& +\frac{R^{2}}{\rho^{2}+w_{1}^{2}+w_{2}^{2}}\left[d \rho^{2}+\rho^{2} d \Omega_{3}^{2}+d w_{1}^{2}+d w_{2}^{2}\right],
\end{aligned}
$$

where $R$ is the AdS radius. Hereafter, we will take a unit of $R=1$ to simplify the following expressions.

Dynamics of the D7-brane is described by the Dirac-Born-Infeld (DBI) action,

$$
S=-T_{7} \int d^{8} \sigma \sqrt{-\operatorname{det}\left[h_{a b}+2 \pi \alpha^{\prime} F_{a b}\right]},
$$

where $T_{7}$ is the tension of the brane, $h_{a b}$ is the induced metric and $F_{a b}=\partial_{a} A_{b}-\partial_{b} A_{a}$ is the $\mathrm{U}(1)$-gauge field strength on the brane. In the $\mathrm{AdS}_{5} \times S^{5}$ spacetime, $\left(t, x, y, z, \rho, \Omega_{3}\right)$ directions are filled with the D7-brane. For simplicity, we consider SQCD with massless quarks in the boundary theory. The brane configuration corresponding to it is given by a trivial solution for the brane position: $w_{1}=w_{2}=0$. To see the consistency of the ansatz $w_{1}=w_{2}=0$, we consider the dynamics of the brane along $w_{1}$ - and $w_{2}$-directions. In the DBI action, there should be U(1)-symmetry associated with the rotational symmetry of the $\left(w_{1}, w_{2}\right)$-plane in the background spacetime: $w \rightarrow e^{i \theta} w$ where $w \equiv w_{1}+i w_{2}$. Thus, in the Lagrangian, $w$ appears as $w w^{*}$ or $\partial w \partial w^{*}$ where $\partial$ represents the derivative by the worldvolume coordinates. In the equations of motion of $w$, every terms are multiplied by $w$ and its derivatives. It follows that $w$ is not sourced by the gauge field $A_{a}$ and $w=0$ is a consistent ansatz. One can also directly check the consistency deriving the equations of motion for $w_{1}$ and $w_{2}$. The induced metric on the D7-brane is simply written as

$$
h_{a b} d \sigma^{a} d \sigma^{b}=\rho^{2}\left[-d t^{2}+d x^{2}+d y^{2}+d z^{2}\right]+\frac{1}{\rho^{2}}\left[d \rho^{2}+\rho^{2} d \Omega_{3}^{2}\right] .
$$

The dynamics of the D7-brane is described only by the gauge field $A_{a}$. We assume the spherical symmetry of $S^{3}$ and translational symmetry in $(x, y, z)$-space. We also set $A_{t}=A_{\rho}=A_{z}=0$, for simplicity, so that there is no baryon number density in the boundary theory. Then, the gauge field $A_{a}$ is written as

$$
\left(2 \pi \alpha^{\prime}\right) A_{a} d \sigma^{a}=a_{x}(t, \rho) d x+a_{y}(t, \rho) d y .
$$

Near the AdS boundary, the rescaled gauge field $\vec{a}=\left(a_{x}, a_{y}\right)$ is expanded as

$$
\vec{a}(t, \rho)=-\int^{t} d t^{\prime} \vec{E}\left(t^{\prime}\right)+\frac{\vec{j}(t)}{2 \rho^{2}}+\frac{\dot{\vec{E}}(t)}{2 \rho^{2}} \ln \left(\frac{\rho}{\rho_{0}}\right)+\cdots, \quad(\rho \rightarrow \infty),
$$


where $\rho_{0}$ is a constant introduced to non-dimensionalize the argument of the logarithmic term. The dot denotes $t$-derivative. The functions $\vec{E}(t)$ and $\vec{j}(t)$ in the series expansion correspond to the electric field $\overrightarrow{\mathcal{E}}(t)$ and current $\vec{J}(t)$ in the boundary theory as

$$
\overrightarrow{\mathcal{E}}(t)=\left(\frac{\lambda}{2 \pi^{2}}\right)^{1 / 2} \vec{E}(t), \quad \vec{J}(t)=\frac{N_{c} \sqrt{\lambda}}{2^{5 / 2} \pi^{3}} \vec{j}(t) .
$$

Note that there is ambiguity in the definition of the electric current $\vec{J}$ when we consider time-dependent electric fields. In eq. (3.5), $\rho$ is normalized by $\rho_{0}$ in the logarithmic term. If we choose a different normalization such as $\ln \left(\rho / \tilde{\rho}_{0}\right)$, the definition of $\vec{j}$ is changed as $\vec{j} \rightarrow \vec{j}+\dot{\vec{E}} \ln \left(\tilde{\rho}_{0} / \rho_{0}\right)$. This ambiguity originates from the ambiguity in the finite local counter term in the DBI action, which should be fixed by a renormalization condition. ${ }^{5}$

In this paper, we will consider a rotating external electric field as in eq. (1.1). The electric field with a constant strength $E$ is parallel to $x$-axis at the initial time $t=0$ and rotating anticlockwise with a frequency $\Omega$ in $(x, y)$-plane. To provide such rotating electric fields conveniently, we introduce the following complex variable,

$$
a(t, \rho) \equiv a_{x}(t, \rho)+i a_{y}(t, \rho) .
$$

For the rotating electric field (1.1), it is also convenient to denote complex electric field and current as

$$
E_{x}+i E_{y}=E e^{i \Omega t}, \quad j_{x}+i j_{y}=j e^{i \Omega t} .
$$

Note that $E$ and $j$ can be complex constants in general. Their magnitudes $|E|$ and $|j|$ describe strength of the electric field and current. Their phases represent the alignment of the electric field and current at $t=0$. Using eqs. (3.3), (3.4) and (3.7), we obtain the action for $a(t, \rho)$ as

$$
S=-T_{7} \Omega_{3} V_{3} \int d t d \rho \rho\left[\rho^{4}-|\dot{a}|^{2}+\rho^{4}\left|a^{\prime}\right|^{2}-\left\{\operatorname{Im}\left(\dot{a} a^{\prime *}\right)\right\}^{2}\right]^{1 / 2},
$$

where $\Omega_{3}=\operatorname{Vol}\left(S^{3}\right)$ and $V_{3}=\int d x d y d z$. For the complex field $a$, the boundary condition (1.1) is written as

$$
\left.a\right|_{\rho=\infty}=\frac{i E}{\Omega} e^{i \Omega t} .
$$

Now, we define a new complex variable $b(t, \rho)$ by factoring out a time-dependent phase factor as

$$
a(t, \rho)=e^{i \Omega t} b(t, \rho) .
$$

For this variable $b$, the above boundary condition becomes time-independent as

$$
\left.b\right|_{\rho=\infty}=\frac{i E}{\Omega} .
$$

\footnotetext{
${ }^{5}$ For the time-dependent gauge field, a logarithmic divergence appears in the on-shell DBI action, which originates from the log term in the asymptotic expansion (3.5). To eliminate the logarithmic divergence from the on-shell action, we need a local counter term proportional to $\log \rho$ [30]. If we change the argument of the $\log$ as $\rho \rightarrow \alpha \rho$, the surface term of the on-shell action is changed. This changes the expression of the electric current.
} 
The action for $b$ is written as

$$
S=-T_{7} \Omega_{3} V_{3} \int d t d \rho \rho\left[\rho^{4}-\left|\left(\partial_{t}+i \Omega\right) b\right|^{2}+\rho^{4}\left|b^{\prime}\right|^{2}-\left\{\operatorname{Im}\left(\left(\partial_{t}+i \Omega\right) b b^{\prime *}\right)\right\}^{2}\right]^{1 / 2} .
$$

In addition to the time-independent boundary condition (3.12), this action does not depend on $t$ explicitly. Thus, we can consistently assume that the variable $b$ does not depend on $t$ : $b(t, \rho)=b(\rho)$. Then, the DBI action becomes

$$
S=-T_{7} V_{4} \int d \rho \mathcal{L}_{0}, \quad \mathcal{L}_{0} \equiv \rho\left[\rho^{4}-\Omega^{2}|b|^{2}+\rho^{4}\left|b^{\prime}\right|^{2}-\Omega^{2}\left\{\operatorname{Re}\left(b b^{\prime *}\right)\right\}^{2}\right]^{1 / 2},
$$

where $V_{4}=\int d t d x d y d z$. This action is invariant under the constant phase rotation: $b \rightarrow$ $e^{i \alpha} b$, where $\alpha$ is an arbitrary real constant. Its Noether charge is given by

$$
q=\frac{\Omega \rho^{6}}{\mathcal{L}_{0}} \operatorname{Im}\left(b^{\prime} b^{*}\right) .
$$

This quantity is conserved along the $\rho$-direction: $d q / d \rho=0$. We will see later that the conserved charge $q$ corresponds to the Joule heating in the boundary theory. The equation of motion for $b$ is given by

$$
\begin{aligned}
b^{\prime \prime}=\frac{1}{4 \rho\left(\rho^{4}-\Omega^{2}|b|^{2}\right)}[ & -12 \rho^{4} b^{\prime}-4 \rho\left(\Omega^{2} b+3 \rho^{3} b^{\prime 2} b^{\prime *}\right) \\
& \left.+\Omega^{2}\left\{4 b^{*} b^{\prime}\left(3 b-\rho b^{\prime}\right)-8 b^{\prime *} b^{2}\right\}+\Omega^{2} b^{\prime}\left(b b^{*}\right)^{\prime 2}\right] .
\end{aligned}
$$

We can obtain the equation of motion for $b^{*}$ by taking the complex conjugate of the above equation. It is remarkable that equations of motion for the brane dynamics reduce to ordinary differential equations (ODEs) even though the electric field is time-dependent. This dramatically simplifies our following analysis. For the reduction to ODEs, it is essential to consider the rotating electric field (1.1). If we consider a linearly (or elliptically) polarized field such as $\vec{E}=E(\cos \Omega t, 0)$ instead, the equations of motion will be given by partial differential equations of $t$ and $\rho$.

\subsection{Boundary conditions at the effective horizon and the AdS boundary}

The equation of motion (3.16) is singular at $\rho=\rho_{c}$ where $\rho_{c}$ satisfies

$$
b\left(\rho_{c}\right)=\frac{\rho_{c}^{2}}{\Omega} e^{i \theta}
$$

where $\theta$ is a real constant. In section 3.3 , we will see that the singular surface $\rho=\rho_{c}$ is an effective event horizon in an effective geometry of the D7-brane. We impose regularity on the solution $b(\rho)$ at $\rho=\rho_{c}$ for physical quantities to be regular. Expanding the solution around $\rho=\rho_{c}$, we obtain the series expansion of the regular solution as

$$
b(\rho)=e^{i \theta} \frac{\rho_{c}^{2}}{\Omega}+e^{i \theta} p\left(\rho-\rho_{c}\right)+\cdots, \quad p \equiv \mathcal{R}_{6}-\sqrt{\mathcal{R}_{4} \mathcal{R}_{9}}+i \sqrt{2 \mathcal{R}_{4}\left(\sqrt{\mathcal{R}_{4} \mathcal{R}_{9}}-\mathcal{R}_{6}\right)}
$$

where we define $\mathcal{R}_{n}=1 / \bar{\rho}_{c}+n \bar{\rho}_{c}$ and $\bar{\rho}_{c}=\rho_{c} / \Omega$. The derivation of the asymptotic solution is summarized in appendix A. Substituting the above expression into eq. (3.15), we can simply write the conserved charge as

$$
q=\rho_{c}^{5}
$$


On the other hand, near the infinity, the asymptotic solution becomes ${ }^{6}$

$$
b(\rho)=\frac{i E}{\Omega}+\frac{j}{2 \rho^{2}}+\frac{i \Omega E}{2 \rho^{2}} \ln \left(\frac{\rho}{\Omega}\right)+\cdots,
$$

where $E$ and $j$ are complex constants introduced in eq. (3.8). Substituting the above expression into eq. (3.15), we have a different expression for the conserved charge as

$$
q=\operatorname{Re}\left(E^{*} j\right)
$$

The constants $E, j \in \mathbf{C}$ correspond to the complex electric field $\mathcal{E}$ and current $J$ in the boundary theory as

$$
\mathcal{E}=\left(\frac{\lambda}{2 \pi^{2}}\right)^{1 / 2} e^{i \Omega t} E, \quad J=\frac{N_{c} \sqrt{\lambda}}{2^{5 / 2} \pi^{3}} e^{i \Omega t} j,
$$

where the real and imaginary parts of $\mathcal{E}$ and $J$ correspond to $x$ - and $y$-components of the electric field and current. The phase $\theta$ in eq. (3.18) is closely connected with the phase of $E$. If one wants to align the electric field with $x$-axis at $t=0$ as in eq. (1.1), one can tune the phase $\theta$ so that $E$ becomes a real value. (See section 3.4 for details.)

It turns out that the Joule heating is given by

$$
Q=\operatorname{Re}\left(\mathcal{E}^{*} J\right)=\frac{N_{c} \lambda}{8 \pi^{4}} \operatorname{Re}\left(E^{*} j\right)=\frac{N_{c} \lambda}{8 \pi^{4}} q
$$

Therefore, the conserved charge $q$ is proportional to the Joule heating. It is worth noting that the conserved current we have seen is nothing but a steady energy-flux along the holographic $\rho$-direction on the D7-brane in the bulk theory. ${ }^{7}$ The rotating electric fields in the boundary theory correspond to the gauge field similar to a usual circular polarized electromagnetic wave in the bulk theory (attend to $(t, x, y, \rho)$-components). Hence, the steady energy-flux like the Poynting flux of the circular polarized electromagnetic wave flows from the AdS boundary into the effective horizon on the D7-brane. The energy injection from the AdS boundary corresponds to the electric power in the boundary theory and the ejection to the effective horizon corresponds to dissipation via the Joule heating. The flow of energy in this system is schematically summarized in figure 3.

\subsection{Effective metric and temperature}

Here, we study an effective metric on the D7-brane [36, 49, 51-54]. The effective metric is defined as

$$
\gamma_{a b}=h_{a b}+\left(2 \pi \alpha^{\prime}\right)^{2} F_{a c} F_{b d} h^{c d} .
$$

Fluctuations on the brane "feel" this effective metric. For example, if one has found the event horizon with respect to $\gamma_{a b}$, we cannot probe inside the horizon using the field on

\footnotetext{
${ }^{6}$ We will normalize $\rho$ by $\rho_{0}=\Omega$ in the logarithmic term throughout this paper.

${ }^{7}$ For the treatment of the heat current in the AdS/CFT correspondence with the flavor brane, see [50].
} 

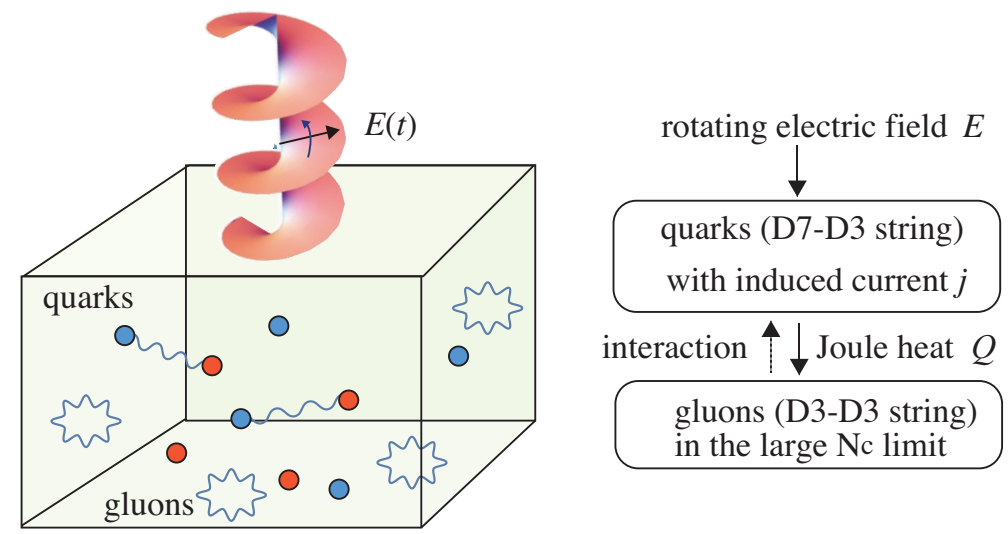

Figure 3. Schematic picture of the energy flow between the degrees of freedom in the system (supersymmetric partners are omitted): the external rotating electric field couples to the quarks and anti-quarks, and induces a current. The gluons mediates a long-range interaction between the quarks while they act as a heat bath stabilizing the NESS.

the brane. From eq. (3.3), (3.4), (3.7) and (3.11), the effective metric is written as

$$
\begin{aligned}
\gamma_{a b} d \sigma^{a} d \sigma^{b}= & -F(\rho) d t^{2}+\frac{2 \Omega \operatorname{Im}\left(b^{\prime} b^{*}\right)}{\rho^{2}} d t d \rho+\frac{1+\left|b^{\prime}\right|^{2}}{\rho^{2}} d \rho^{2} \\
& +\frac{\rho^{2}}{4}\left(b^{\prime} e_{-}+b^{\prime *} e_{+}\right)^{2}+\frac{\Omega^{2}}{4 \rho^{2}}\left(b e_{-}+b^{*} e_{+}\right)^{2} \\
& +F(\rho)\left(e_{1}^{2}+e_{2}^{2}\right)+\rho^{2} e_{3}^{2}+d \Omega_{3}^{2},
\end{aligned}
$$

where

$$
F(\rho)=\frac{\rho^{4}-\Omega^{2}|b|^{2}}{\rho^{2}} .
$$

We have also defined 1 -forms $\left(e_{1}, e_{2}, e_{3}\right)$ and $e_{ \pm}$as

$$
\left(\begin{array}{l}
e_{1} \\
e_{2} \\
e_{3}
\end{array}\right)=\left(\begin{array}{ccc}
\cos \Omega t & \sin \Omega t & 0 \\
-\sin \Omega t & \cos \Omega t & 0 \\
0 & 0 & 1
\end{array}\right)\left(\begin{array}{l}
d x \\
d y \\
d z
\end{array}\right), \quad e_{ \pm}=e_{1} \pm i e_{2} .
$$

Note that $F(\rho)$ becomes zero at $\rho=\rho_{c}$. This implies that $\rho=\rho_{c}$ is the event horizon with respect to the effective metric $\gamma_{a b}{ }^{8}$

\footnotetext{
${ }^{8}$ Let us consider past-directed null geodesics starting from the AdS boundary for the effective metric $\gamma_{a b}$. Any tangent vector of the null geodesics must satisfy $\gamma_{a b} \frac{d \sigma^{a}}{d s} \frac{d \sigma^{b}}{d s}=0$, where $s$ is an affine parameter. Since, from the explicit form of the effective metric (3.25), the norm becomes a sum of squares in terms of components of the tangent vector other than $(t, \rho)$-directions, we have

$$
-F(\rho)\left(\frac{d t}{d s}\right)^{2}+\frac{2 \Omega \operatorname{Im}\left(b^{\prime} b^{*}\right)}{\rho^{2}} \frac{d t}{d s} \frac{d \rho}{d s}+\frac{1+\left|b^{\prime}\right|^{2}}{\rho^{2}}\left(\frac{d \rho}{d s}\right)^{2} \leq 0 .
$$

This means that arbitrary null geodesics on the whole brane worldvolume can be projected to timelike curves on the $(t, \rho)$-subspace, or they become null only if the tangent vector of the null geodesics has just the $(t, \rho)$-components. Considering the $(t, \rho)$-subspace alone, the locus $\rho=\rho_{c}$ where $F(\rho)=0$ is the event horizon, namely any past-directed timelike or null curves on the $(t, \rho)$-subspace cannot enter $\rho \leq \rho_{c}$. As a result, the past-directed null geodesics on the whole brane worldvolume cannot enter the region $\rho \leq \rho_{c}$ and the surface $\rho=\rho_{c}$ is the event horizon with respect to the effective metric $\gamma_{a b}$.
} 
It turns out that while the coefficients in the effective metric (3.25) are independent of the time-coordinate $t$ and depend only on $\rho$, the basis 1-forms $e_{1}$ and $e_{2}$ explicitly depend on $t$. This means that the effective metric is time-dependent and $\partial_{t}$ is not a stationary Killing vector field with respect to $\gamma_{a b}$. However, on this metric there remains a time-like Killing vector field defined by $\chi^{a} \partial_{a}=\partial_{t}+\Omega\left(x \partial_{y}-y \partial_{x}\right)$. Indeed, since we can explicitly confirm that the basis 1 -forms satisfy $\mathcal{L}_{\chi} e_{1}=0$ and $\mathcal{L}_{\chi} e_{2}=0$, the effective metric $\gamma_{a b}$ also satisfies $\mathcal{L}_{\chi} \gamma_{a b}=0$. This Killing vector is a generator of symmetry for other fields such as the worldvolume gauge field and the induced metric as well as the effective metric, that is, $\mathcal{L}_{\chi} a_{a}=0$ and $\mathcal{L}_{\chi} h_{a b}=0$. The conserved current previously shown in (3.15) is nothing but a consequence of this symmetry, because a constant time translation generates a constant rotation of the alignment of the gauge fields $\left(a_{x}, a_{y}\right)$ which corresponds to a constant phase shift for $b(\rho)$. The norm of the Killing vector with respect to the effective metric becomes

$$
\begin{aligned}
\gamma_{a b} \chi^{a} \chi^{b}= & -F(\rho)+\frac{\Omega^{2}\left(x^{2}+y^{2}\right)}{2 \rho^{2}}\left(2 \rho^{2} F(\rho)+\rho^{4}\left|b^{\prime}\right|^{2}+\Omega^{2}|b|^{2}\right) \\
& -\frac{\Omega^{2}}{2 \rho^{2}} \operatorname{Re}\left[e^{2 i \Omega t}(x-i y)^{2}\left(\Omega^{2} b^{2}+\rho^{4} b^{2}\right)\right] .
\end{aligned}
$$

On the surface $\rho=\rho_{c}, \chi^{a}$ is not null vector except for $x=0$ and $y=0$. The surface $\rho=\rho_{c}$ is not the Killing horizon generated by the Killing vector $\chi$. However, because the system has translational invariance along $x$ - and $y$-directions, even at any $x=x_{0}$ and $y=y_{0}$ there exist other Killing vectors which become null at $\rho=\rho_{c}$ such as $\tilde{\chi}^{a} \partial_{a}=\partial_{t}+\Omega\left[\left(x-x_{0}\right) \partial_{y}-\left(y-y_{0}\right) \partial_{x}\right]$. Although null Killing vectors exist everywhere on the event horizon, these Killing vectors do not belong to a single Killing vector field.

The effective metric is explicitly regular at $\rho=\rho_{c}$. The Hawking temperature is computed from the surface gravity $\kappa$ on the event horizon as

$$
T_{H}=\frac{\kappa}{2 \pi}=\frac{2 \rho_{c}-\Omega \operatorname{Re} p}{2 \pi \operatorname{Im} p}
$$

where $p$ is given in eq. (3.18). Here, the surface gravity we have defined above is different from the usual definition in the sense that the event horizon of the effective geometry is not a Killing horizon. However, since the null Killing vectors associated with the event horizon exist as we mentioned, we can define a surface gravity on the event horizon with respect to these Killing vectors. For example, we have $\chi^{a} D_{a} \chi^{b}=\kappa \chi^{b}$ on the event horizon for a Killing vector $\chi^{a}$ satisfying $\left.\chi^{a} \chi^{b} \gamma_{a b}\right|_{\rho=\rho_{c}}=0$. Moreover, if we restrict our attention to the $(t, \rho)$ subspace, the event horizon $\rho=\rho_{c}$ can be regarded as the Killing horizon on the subspace because the subspace is time-independent. We can also obtain the above surface gravity $\kappa$ in the same way as the surface gravity with the Killing horizon for the $(t, \rho)$-subspace. Quantum fluctuations which can reach the AdS boundary from neighborhood of the event horizon are dominated by the $s$-wave modes, which are homogeneous along the spatial directions tangent to the horizon. In other words, null geodesics with no components other than $(t, \rho)$ can mainly arrive at the boundary. The Hawking temperature of the current system can be characterized by this surface gravity.

In eq. (3.19), the Joule heating $q$ is expressed by the function of $\rho_{c}$ as $q=\rho_{c}^{5}$. Thus, replacing $\rho_{c}$ by $q^{1 / 5}$ in eq. (3.29), we can express the Joule heating as a function of $T_{H}$. 


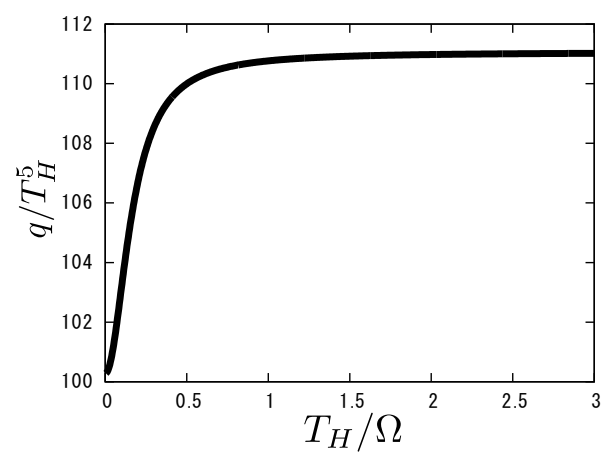

Figure 4. Joule heating $q$ as a function of Hawking temperature $T_{H}$.

For $T_{H} \gg \Omega$ and $T_{H} \ll \Omega$, the Joule heating $q$ is simply written as

$$
q=\left(\frac{4 \pi}{5} T_{H}\right)^{5}, \quad\left(T_{H} \ll \Omega\right), \quad q=\left(\frac{2 \pi}{\sqrt{6}} T_{H}\right)^{5}, \quad\left(T_{H} \gg \Omega\right) .
$$

Figure 4 shows the functional form of $q\left(T_{H}\right)$ interpolating two limits. For the visibility, we have divided $q$ by $T_{H}^{5}$ at the vertical axis in this figure.

We introduce coordinates $\left(\tau, \rho_{*}\right)$ as

$$
d \tau=d t-\frac{\Omega \operatorname{Im}\left(b^{\prime} b^{*}\right)}{\rho^{2} F(\rho)} d \rho, \quad d \rho_{*}=\frac{\mathcal{L}_{0}}{\rho^{3} F(\rho)} d \rho .
$$

The range of the tortoise coordinate $\rho_{*}$ is $-\infty<\rho_{*} \leq 0$. In term of these coordinates, $(t, \rho)$-part of the effective metric can be diagonalized as

$$
\begin{aligned}
\gamma_{a b} d \sigma^{a} d \sigma^{b}= & F(\rho)\left(-d \tau^{2}+d \rho_{*}^{2}\right)+\frac{\rho^{2}}{4}\left(b^{\prime} e_{-}+b^{\prime *} e_{+}\right)^{2}+\frac{\Omega^{2}}{4 \rho^{2}}\left(b e_{-}+b^{*} e_{+}\right)^{2} \\
& +F(\rho)\left(e_{1}^{2}+e_{2}^{2}\right)+\rho^{2} e_{3}^{2}+d \Omega_{3}^{2} .
\end{aligned}
$$

In section 4 , we will find that the new coordinate $\left(\tau, \rho_{*}\right)$ are better suited to describe the perturbation of the background solution $b(\rho)$.

\subsection{Physical quantities of the holographic Floquet state}

Here, we determine the $b(\rho)$ numerically and evaluate the physical quantities in the boundary theory. Note that our system is invariant under the scaling symmetry:

$$
\begin{aligned}
& \rho \rightarrow \lambda \rho, \quad t \rightarrow t / \lambda, \quad \Omega \rightarrow \lambda \Omega, \quad b \rightarrow \lambda b, \\
& E \rightarrow \lambda^{2} E, \quad j \rightarrow \lambda^{3} j, \quad q \rightarrow \lambda^{5} q, \quad T_{H} \rightarrow \lambda T_{H},
\end{aligned}
$$

where $\lambda$ is a non-zero constant. Using the scaling symmetry, we will set $\Omega=1$ in our numerical calculation. We solve eq. (3.16) from $\rho=\rho_{c}+\delta$ to $\rho=\rho_{\max }$, where we typically set $\delta=1.0 \times 10^{-6}$ and $\rho_{\max }=100$. At the inner boundary $\rho=\rho_{c}+\delta$, we impose the boundary condition in eq. (3.18). At first, we set $\theta=0$ tentatively and obtain a solution $b_{0}(\rho)$. From numerical values of $b_{0}\left(\rho_{\max }\right)$ and $b_{0}^{\prime}\left(\rho_{\max }\right)$, we read off $E_{0}, j_{0} \in \mathbf{C}$ using eq. (3.20). To make $E$ real, we choose $\theta=-\arg \left(E_{0}\right)$ and obtain a desirable solution 


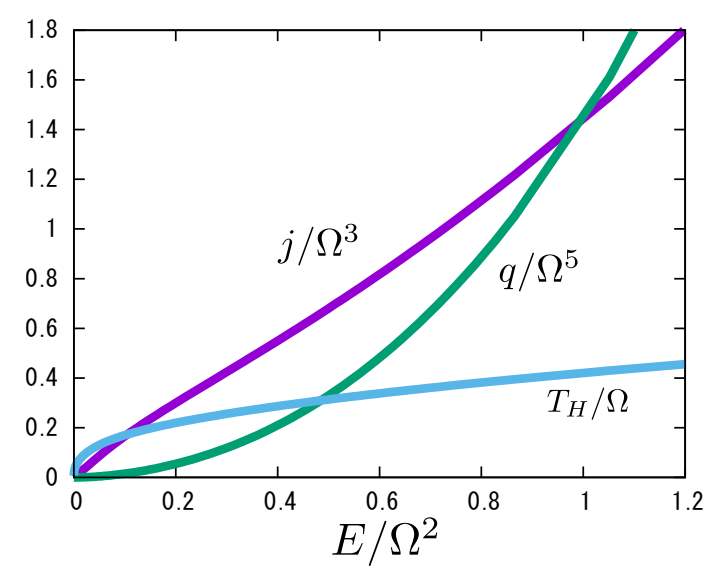

(a) Varying $|E|$ for fixed $\Omega$

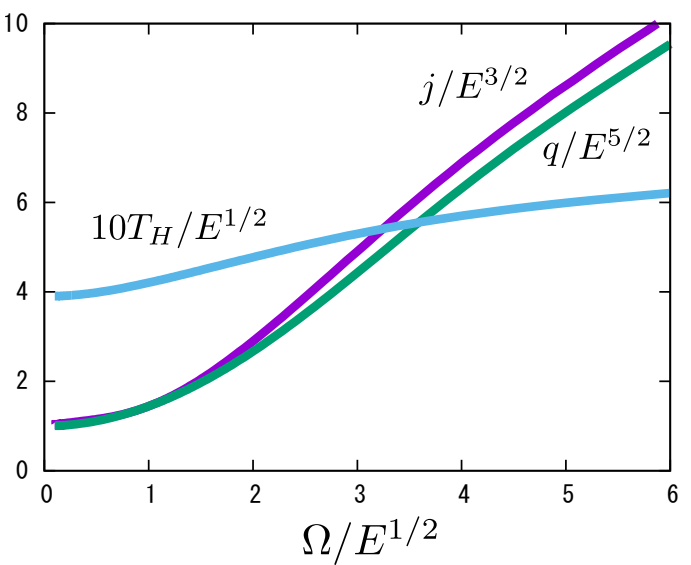

(b) Varying $\Omega$ for fixed $E$

Figure 5. Electric current, Joule heating and Hawking temperature. They are increasing functions of $E$ and $\Omega$ for fixed $\Omega$ and $E$, respectively. In the right figure, we multiply 10 to Hawking temperature $T_{H}$ for visibility.

$b(\rho)=e^{i \theta} b_{0}(\rho)$, which satisfies the boundary condition (1.1). We repeat above procedure for several $\rho_{c}$ and obtain $E\left(\rho_{c}\right) \in \mathbf{R}$ and $j\left(\rho_{c}\right) \in \mathbf{C}$. We can also compute the Joule heating $q$ and Hawking temperature $T_{H}$ as functions of $\rho_{c}$ from eqs. (3.21) and (3.29). In figure $5(\mathrm{a})$, we show scaling invariant quantities $|j| / \Omega^{3}, q / \Omega^{5}$ and $T_{H} / \Omega$ as functions of $E / \Omega^{2}$ regarding $\rho_{c}$ as a parameter. On the other hand, in figure $5(\mathrm{~b})$, we show the same results in different normalization: $|j| / E^{3 / 2}, q / E^{5 / 2}$ and $T_{H} / E^{1 / 2}$ vs $\Omega / E^{1 / 2}$. Although these figures are essentially same, they are convenient to see two kinds of physical process: varying $E$ for fixed $\Omega$ and varying $\Omega$ for fixed $E$. In the left figure, we see that $|j|, q$ and $T_{H}$ increase as functions of $E$ for fixed $\Omega$ as one can expect. In the right figure, however, we find non-trivial feature: $|j|, q$ and $T_{H}$ are also increasing functions of the frequency $\Omega$ for fixed $E$. It follows that, the more quickly we rotate the electric field, the more effectively the electric current is generated. The vertical axis $\Omega=0$ in the right figure corresponds to the direct current (DC) electric field, or more precisely a static electric field. The D3/D7 systems with the DC electric fields were studied in refs. [30-33, 36]. In our notation, the electric current has been given by $j=E^{3 / 2}, q=E^{5 / 2}$ and $T_{H}=(6 E)^{1 / 2} /(2 \pi) \simeq 0.39 E^{1 / 2}$. Therefore, our results for the rotating electric fields can consistently reproduce results for the static electric fields in the limit $\Omega \rightarrow 0$.

\section{Hall effect of the holographic Floquet state}

\subsection{Conductivities}

In this section, we study the linear response of the holographic Floquet state against probe $\mathrm{AC}$ and DC electric fields. We apply the probe $\mathrm{AC}$ electric field $\mathrm{as}^{9}$

$$
\vec{\varepsilon}(t)=\vec{\varepsilon}_{\omega} e^{-i \omega t} .
$$

\footnotetext{
${ }^{9}$ For perturbation theory, we will use the vector notation instead of the complex notation.
} 
By the probe electric field, the gauge field on the brane is perturbed as $\vec{a} \rightarrow \vec{a}+\delta \vec{a}$. The boundary condition for the perturbation of gauge field $\delta \vec{a}$ becomes

$$
\left.\delta \vec{a}\right|_{\rho=\infty}=-\frac{i \vec{\varepsilon}_{\omega}}{\omega} e^{-i \omega t} .
$$

For the perturbation of $\vec{b}$, the boundary condition is written as

$$
\left.\delta \vec{b}\right|_{\rho=\infty}=\left.\left(\begin{array}{cc}
\cos \Omega t & \sin \Omega t \\
-\sin \Omega t & \cos \Omega t
\end{array}\right) \delta \vec{a}\right|_{\rho=\infty}=-\frac{i}{\omega}\left(M_{-} e^{-i \omega_{-} t}+M_{+} e^{-i \omega_{+} t}\right) \vec{\varepsilon}_{\omega},
$$

where $\omega_{ \pm}=\omega \pm \Omega$ and $\boldsymbol{M}_{ \pm}$is a constant matrix defined by

$$
\boldsymbol{M}_{ \pm}=\frac{1}{2}\left(\begin{array}{cc}
1 & \pm i \\
\mp i & 1
\end{array}\right)
$$

The perturbation equation for $\delta \vec{b}$ is written as

$$
\left[\partial_{\tau}^{2}-\partial_{\rho_{*}}^{2}+\boldsymbol{A}(\rho) \partial_{\tau}+\boldsymbol{B}(\rho) \partial_{\rho_{*}}+\boldsymbol{C}(\rho)\right] \delta \vec{b}=0,
$$

where we have used coordinates $\left(\tau, \rho_{*}\right)$ defined in eq. (3.31) and $\boldsymbol{A}, \boldsymbol{B}$ and $\boldsymbol{C}$ are $\rho$ dependent $2 \times 2$ matrices. Their explicit expressions are summarized in appendix B. From eq. (4.3), fluctuations with frequencies $\omega_{+}$and $\omega_{-}$are induced by the boundary conditions. Thus, $\delta \vec{b}$ is expanded as

$$
\delta \vec{b}=\vec{\beta}_{+}(\rho) e^{-i \omega_{+} t}+\vec{\beta}_{-}(\rho) e^{-i \omega_{-} t} .
$$

From eq. (4.5), we obtain decoupled equations for $\vec{\beta}_{ \pm}$as

$$
\left[\frac{d^{2}}{d \rho_{*}^{2}}-\boldsymbol{B}(\rho) \frac{d}{d \rho_{*}}+\omega_{ \pm}^{2}+i \omega_{ \pm} \boldsymbol{A}(\rho)-\boldsymbol{C}(\rho)\right] \vec{\beta}_{ \pm}=0 .
$$

Near the AdS boundary $\rho_{*}=0$, asymptotic forms of $\vec{\beta}_{ \pm}$are

$$
\vec{\beta}_{ \pm}(\rho)=\vec{\beta}_{ \pm}^{(0)}+\vec{\beta}_{ \pm}^{(2)} \rho_{*}^{2}-\frac{1}{2}\left(\omega_{ \pm}^{2}+\Omega^{2}-2 i \Omega \omega_{ \pm} \boldsymbol{\epsilon}\right) \vec{\beta}_{ \pm}^{(0)} \rho_{*}^{2} \ln \left(-\Omega \rho_{*}\right)+\cdots
$$

where $\vec{\beta}_{ \pm}^{(0)}$ and $\vec{\beta}_{ \pm}^{(2)}$ are constant vectors and $\boldsymbol{\epsilon}$ is the anti-symmetric matrix with $\epsilon_{12}=1$. From the boundary condition eq. (4.3), the leading term must be

$$
\vec{\beta}_{ \pm}^{(0)}=-\frac{i}{\omega} M_{ \pm} \vec{\varepsilon}_{\omega}
$$

On the other hand, we impose the ingoing wave boundary condition at the horizon:

$$
\vec{\beta}_{ \pm} \propto e^{-i \omega_{ \pm} \rho_{*}}, \quad\left(\rho \rightarrow \rho_{c}\right) .
$$

The asymptotic solution of $\delta \vec{b}$ near the horizon is studied in appendix B. From eq. (4.8), we can also write down the asymptotic solution of the original gauge field $\delta \vec{a}$ near the AdS boundary. Using eq. (3.5), we can read off the electric current induced by the probe electric field as

$$
\delta \vec{j}_{\omega}=2 e^{-i \omega t}\left[\left(\boldsymbol{M}_{+} \vec{\beta}_{+}^{(2)}+\boldsymbol{M}_{-} \vec{\beta}_{-}^{(2)}\right)+\boldsymbol{M}_{+} \vec{\beta}_{-}^{(2)} e^{2 i \Omega t}+\boldsymbol{M}_{-} \vec{\beta}_{+}^{(2)} e^{-2 i \Omega t}\right] .
$$


Even though we have introduced the monochromatic AC electric field with the frequency $\omega$ as in eq. (4.2), three modes are induced in the electric current, whose frequencies are given by $\omega$ and $\omega \pm 2 \Omega$. This is known as the heterodyning effect characteristic to periodically driven Floquet systems [46]. Thus, we can define three kinds of conductivities $\boldsymbol{\sigma}$ and $\boldsymbol{\sigma}^{ \pm}$as $^{10}$

$$
\delta \vec{j}_{\omega}=\left[\boldsymbol{\sigma}(\omega) e^{-i \omega t}+\boldsymbol{\sigma}^{+}(\omega) e^{-i(\omega+2 \Omega) t}+\boldsymbol{\sigma}^{-}(\omega) e^{-i(\omega-2 \Omega) t}\right] \vec{\varepsilon}_{\omega},
$$

where $\boldsymbol{\sigma}$ 's are $2 \times 2$ complex matrices. Conductivities $\boldsymbol{\sigma}$ and $\boldsymbol{\sigma}^{ \pm}$have $4 \times 3=12$ complex components. However, they are not independent but there are the following 8 relations:

$$
\begin{aligned}
& \sigma_{x x}=\sigma_{y y}, \quad \sigma_{x y}=-\sigma_{y x}, \\
& \sigma_{x x}^{ \pm}=-\sigma_{y y}^{ \pm}, \quad \sigma_{x y}^{ \pm}=\sigma_{y x}^{ \pm}, \quad \sigma_{y x}^{ \pm}= \pm i \sigma_{x x}^{ \pm} .
\end{aligned}
$$

We give a proof of these relations in appendix C. Therefore, independent complex degrees of freedom of conductivities are $12-8=4$. We will take $\sigma_{x x}, \sigma_{x y}$ and $\sigma_{x x}^{ \pm}$as the independent components. Taking the complex conjugate of eq. (4.12) and using reality conditions $\delta j_{-\omega}=\delta j_{\omega}^{*}$ and $\varepsilon_{-\omega}=\varepsilon_{\omega}^{*}$, we obtain

$$
\boldsymbol{\sigma}(-\omega)=(\boldsymbol{\sigma}(\omega))^{*}, \quad \boldsymbol{\sigma}^{ \pm}(-\omega)=\left(\boldsymbol{\sigma}^{\mp}(\omega)\right)^{*} .
$$

So, we will consider the conductivities only in $\omega \geq 0$.

\subsection{Hall effect}

Here, we study the DC conductivity in the holographic Floquet state obtained in section 3. In the previous subsection, we have considered the perturbation of the gauge field in the frequency domain. However, it is not directly applicable for the probe DC electric field since eq. (4.2) becomes singular at $\omega=0$. Therefore, we take the time-domain approach to evaluate the DC conductivities. We will find that the DC conductivities computed in the time domain will coincide with the $\mathrm{AC}$ conductivities computed in the frequency domain when taking the limit of $\omega \rightarrow 0$.

We consider a quench-type function for the probe electric field as

$$
\vec{\varepsilon}(t)=\vec{\varepsilon}_{f} f(t), \quad f(t) \equiv \begin{cases}0 & (t<0) \\ {\left[t-\frac{\Delta t}{2 \pi} \sin (2 \pi t / \Delta t)\right] / \Delta t} & (0 \leq t \leq \Delta t) . \\ 1 & (t>\Delta t)\end{cases}
$$

Here, $\vec{\varepsilon}_{f}$ is a final value of the electric field and $\Delta t$ is a duration of the quench. The boundary condition at the AdS boundary for $\delta \vec{b}$ becomes

$$
\left.\delta \vec{b}\right|_{\rho=\infty}=-\left(\begin{array}{cc}
\cos \Omega t & \sin \Omega t \\
-\sin \Omega t & \cos \Omega t
\end{array}\right) \int_{0}^{t} d t^{\prime} \vec{\varepsilon}\left(t^{\prime}\right) .
$$

\footnotetext{
${ }^{10}$ Again there is an ambiguity in the electric current $\delta \vec{j} \rightarrow \delta \vec{j}+\alpha \dot{\vec{\varepsilon}}$, where $\alpha$ is a constant. This ambiguity appears in the conductivity as $\boldsymbol{\sigma} \rightarrow \boldsymbol{\sigma}-i \alpha \omega$. Thus, only $\operatorname{Im} \sigma_{x x}$ and $\operatorname{Im} \sigma_{y y}$ are affected by the ambiguity in the conductivities.
} 
The numerical method to solve the perturbation equations in the time domain is summarized in appendix D. From the numerical solution of $\delta \vec{b}(t, \rho)$, we can compute the perturbation of the original gauge field, $\delta \vec{a}(t, \rho)$. The asymptotic form of $\delta \vec{a}(t, \rho)$ near the AdS boundary is written as

$$
\delta \vec{a}(t, \rho)=-\int_{0}^{t} d t^{\prime} \vec{\varepsilon}\left(t^{\prime}\right)+\frac{\delta \vec{j}(t)}{2 \rho^{2}}+\frac{\dot{\vec{\varepsilon}}(t)}{2 \rho^{2}} \ln \left(\frac{\rho}{\Omega}\right),
$$

where $\delta \vec{j}(t)$ is the perturbation of the electric current. From the numerical solution, we read off the electric current induced by the probe electric field $\vec{\varepsilon}$. Taking the limit of $\omega \rightarrow 0$ in eq. (4.12), we obtain the electric current at late time as $\delta \vec{j}=\left[\boldsymbol{\sigma}(0)+\boldsymbol{\sigma}^{+}(0) e^{-2 i \Omega t}+\right.$ $\left.\boldsymbol{\sigma}^{-}(0) e^{2 i \Omega t}\right] \vec{\varepsilon}_{f}$. Note that, from eq. (4.14), $\boldsymbol{\sigma}(0)$ becomes a real matrix and $\boldsymbol{\sigma}^{+}(0)$ is the complex conjugate of $\boldsymbol{\sigma}^{-}(0)$. Combining them with eq. (4.13), we can take the independent components of the conductivities as $\sigma_{x x}(0), \sigma_{x y}(0) \in \mathbf{R}$ and $\sigma_{x x}^{+}(0) \in \mathbf{C}$ (four real components). In the actual numerical calculation, we set $\vec{\varepsilon}_{f}=(1,0)$. Then, the late time expression of the current is written as

$$
\delta \vec{j}=\left(\begin{array}{c}
\sigma_{x x}(0) \\
-\sigma_{x y}(0)
\end{array}\right)+2\left(\begin{array}{c}
\operatorname{Re} \sigma_{x x}^{+}(0) \\
-\operatorname{Im} \sigma_{x x}^{+}(0)
\end{array}\right) \cos 2 \Omega t+2\left(\begin{array}{c}
\operatorname{Im} \sigma_{x x}^{+}(0) \\
\operatorname{Re} \sigma_{x x}^{+}(0)
\end{array}\right) \sin 2 \Omega t .
$$

In figure 6 , we show the time dependence of $\delta j_{x}$ and $\delta j_{y}$ for $E / \Omega^{2}=0.498$ and $\Omega \Delta t=10$ as an example. Note that, even though the probe DC electric field $\vec{\varepsilon}$ does not have oscillating components at late time, we obtain oscillating response currents because of the background rotating electric field. We can find that the time-average of the $\delta j_{y}$ at the late time has a non-zero negative value. This is nothing but the Hall effect induced by the rotating external electric field. The oscillations of $\delta j_{x}$ and $\delta j_{y}$ come from $\cos 2 \Omega t$ and $\sin 2 \Omega t$ in eq. (4.18). When we apply the probe DC electric field along $x$-direction, we obtain the Hall current towards $(-y)$-direction. (In other wards, $\left.\sigma_{x y}\right|_{\omega=0}$ is positive.) This is one of the predictions on the strongly coupled version of the "Floquet Weyl semimetal". In the non-interacting Floquet Weyl semimetals with broken time reversal symmetry, it is known that the Hall effect exists due to the "Berry curvature effect" since the Weyl points act as monopoles in the momentum space [20]. Our observation suggests that this Hall effect survives in the strong coupling limit, where now a clear single body picture as in the non-interacting case is lacking.

In figure 7, we show the DC Hall conductivity $\left.\sigma_{x y}\right|_{\omega=0}$ as the function of background parameters by fitting the numerical result of $\delta \vec{j}(t)$ to eq. (4.18): (a) $\left.\sigma_{x y}\right|_{\omega=0} / \Omega$ vs $E / \Omega^{2}$, and (b) $\left.\sigma_{x y}\right|_{\omega=0} / E^{1 / 2}$ vs $\Omega / E^{1 / 2}$. As expected, we see that the Hall response is zero when the rotating electric field is absent $(E=0)$, and then it sharply increases when we turn the field on. However, we find that the Hall response is not a monotonic function of the field but has a maximum. When we vary $E$ for a fixed $\Omega$, the DC Hall conductivity has a maximum value $\left.\sigma_{x y}\right|_{\omega=0}=0.0696 \Omega$ at $E=0.639 \Omega^{2}$. On the other hand, when we vary $\Omega$ for a fixed $E$, it has a maximum value $\left.\sigma_{x y}\right|_{\omega=0}=0.153 E^{1 / 2}$ at $\Omega=4.39 E^{1 / 2}$. What is the reason that the Hall conductivity peaks out and start to decrease? The large $E$ limit is also the small $\Omega$ limit where the electric field is rotating adiabatically slowly. We do not expect to have a Hall effect in a static electric field background, i.e., at $\Omega=0$. Then why should we expect 


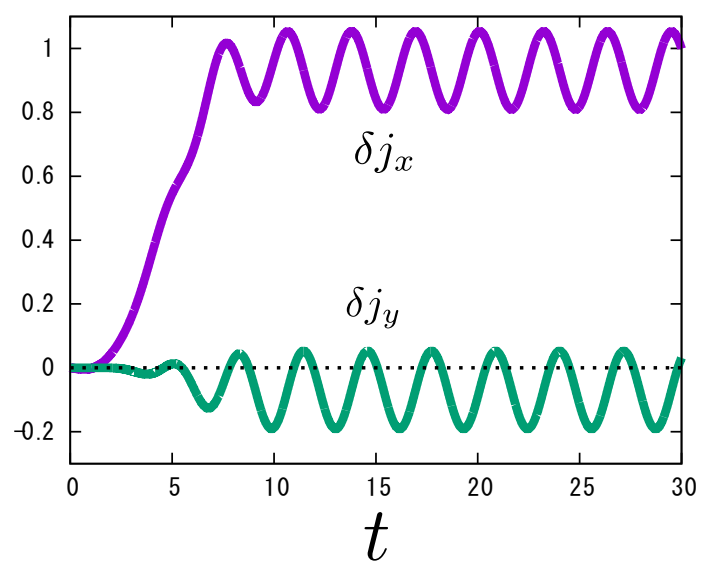

Figure 6. Time dependence of the electric currents for $E=0.498, \Omega=1, \Delta t=10$ and $\vec{\varepsilon}_{f}=(1,0)$. The time-average of the $\delta j_{y}$ at the late time has a non-zero value.

an peak at $E=0.639 \Omega^{2}$ ? Currently, we can only make speculations, but it seems that the non-interacting result provides a hint. In section 2 , we have shown that in the weak coupling limit, the Weyl points, which causes the Hall response, vanishes at $E=0.5 \Omega^{2}$. This occurs through a pair annihilation of Weyl points with the new Weyl points that emerges from Floquet side bands [20]. The peak in the holographic model $\left(E=0.639 \Omega^{2}\right)$ is not far away from $E=0.5 \Omega^{2}$, so it might be caused by this disappearance of the Weyl nodes in the Floquet spectrum. Another possibility is through destruction of the Weyl band due to interaction. For example, in ref. [48], it was pointed out that a gap may open in a single Weyl point when there are fermion-fermion interactions.

We show the diagonal conductivity $\sigma_{x x}$ in the insets of these figures 7 . They are increasing functions of $E$ and $\Omega$ for fixed $\Omega$ and $E$, respectively. The increase can be understood by heating. The rotating electric field is creating quarks-anti-quark pairs through pair production, i.e., $\gamma \rightarrow q \bar{q}$ process. The surrounding gluons stabilizes this process and we are left with a nonequilibrium steady state distribution with an effective temperature (figure 5) increasing as the field is increase. In semimetals, the conductivity $\sigma_{x x}$ is a slowly increasing function of temperature, which seems to explain what we observe in our calculations. Larger $E$ strengthens the heating while larger $\Omega$ increases the energy absorbed in each pair creation processes. Let us note that in some situations the conductivity can drop by increasing the field strength. This can happen when the field generates a gap, which was demonstrated in a non-interacting problem of a $(2+1)$ dimensional Dirac system in rotating electric fields [55].

The other conductivities $\boldsymbol{\sigma}^{ \pm}$that we have also studied are summarized in appendix E.

\subsection{Optical Hall effect}

We study the conductivities for AC probe electric fields $(\omega>0)$ using the frequencydomain approach shown in section 4.1. Technical details are as follows. We solve eq. (4.7) from the effective horizon $\left(\rho_{*}=-\infty\right)$ to the AdS boundary $\left(\rho_{*}=0\right)$ using the fourth order Runge-Kutta method. At the effective horizon, we impose the boundary conditions 


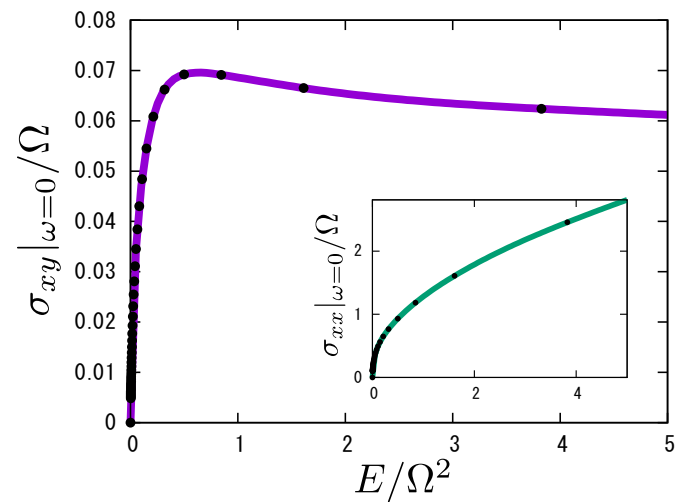

(a) Varying $E$ for fixed $\Omega$

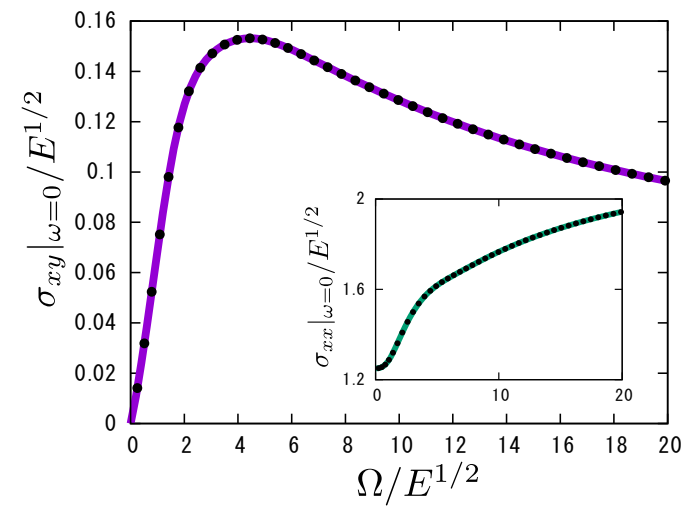

(b) Varying $\Omega$ for fixed $E$

Figure 7. The DC Hall conductivity of the holographic Floquet state. In the left and right figures, parameter are nondimensionalized by $\Omega$ and $E$, respectively. They have maximum values at $\left(E / \Omega^{2},\left.\sigma_{x y}\right|_{\omega=0} / \Omega\right)=(0.639,0.0696)$ [left] and $\left(\Omega / E^{1 / 2},\left.\sigma_{x y}\right|_{\omega=0} / E^{1 / 2}\right)=(4.39,0.153)$ [right]. The insets of these figures are for $\sigma_{x x}$.

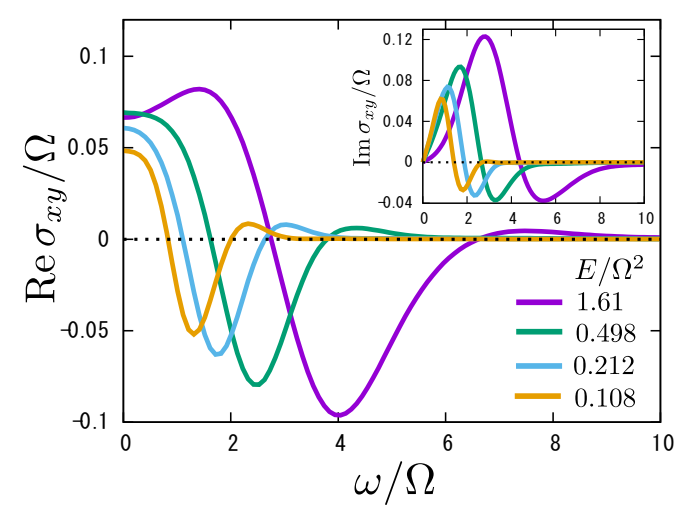

(a) Varying $E$ for fixed $\Omega$

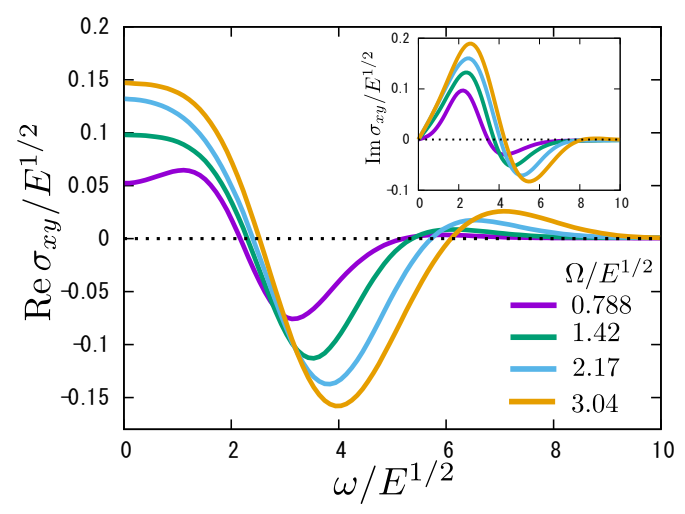

(b) Varying $\Omega$ for fixed $E$

Figure 8. The optical Hall coefficients against the probe AC frequency $\omega$. Curves in the left (right) figure correspond to several $E(\Omega)$ for a fixed $\Omega(E)$.

$\vec{\beta}_{ \pm}=\vec{c}_{ \pm} e^{-i \omega_{ \pm} \rho_{*}}$ as in eq. (4.10) where $\vec{c}_{ \pm}$are constant vectors. We solve the perturbation equation twice for two initial conditions: $\vec{c}_{ \pm}=(1,0)$ and $(0,1)$. From asymptotic forms of the numerical solutions, we read off $\vec{\beta}_{ \pm}^{(0)}$ and $\vec{\beta}_{ \pm}^{(2)}$ defined in eq. (4.8). Because of the linearity, we obtain complex $2 \times 2$ matrices $P_{ \pm}$and $Q_{ \pm}$defined by $\vec{\beta}_{ \pm}^{(0)}=P_{ \pm} \vec{c}_{ \pm}$and $\vec{\beta}_{ \pm}^{(2)}=Q_{ \pm} \vec{c}_{ \pm}$. Then, we can express $\vec{\beta}_{ \pm}^{(2)}$ by $\vec{\beta}_{ \pm}^{(0)}$ as $\vec{\beta}_{ \pm}^{(2)}=Q_{ \pm} P_{ \pm}^{-1} \vec{\beta}_{ \pm}^{(0)}$. Using eq. (4.9), we have $\vec{\beta}_{ \pm}^{(2)}=-(i / \omega) Q_{ \pm} P_{ \pm}^{-1} \boldsymbol{M}_{ \pm} \vec{\varepsilon}_{\omega}$. Substituting this into eq. (4.11) and reading off coefficients of $e^{-i \omega t}$ and $e^{-i(\omega \pm 2 \Omega) t}$, we obtain the AC conductivities $\boldsymbol{\sigma}$ and $\boldsymbol{\sigma}^{ \pm}$.

In figure 8, we show the optical Hall conductivities $\sigma_{x y}$ against the probe AC frequency $\omega$. Curves in the left (right) figure correspond to several $E(\Omega)$ for a fixed $\Omega(E)$. In the DC limit $\omega \rightarrow 0$, we can check that $\operatorname{Re} \sigma_{x y}$ approaches the DC Hall coefficient obtained in figure 7 . In the both figures, $\operatorname{Re} \sigma_{x y}$ have peaks in the negative region. The peaks 


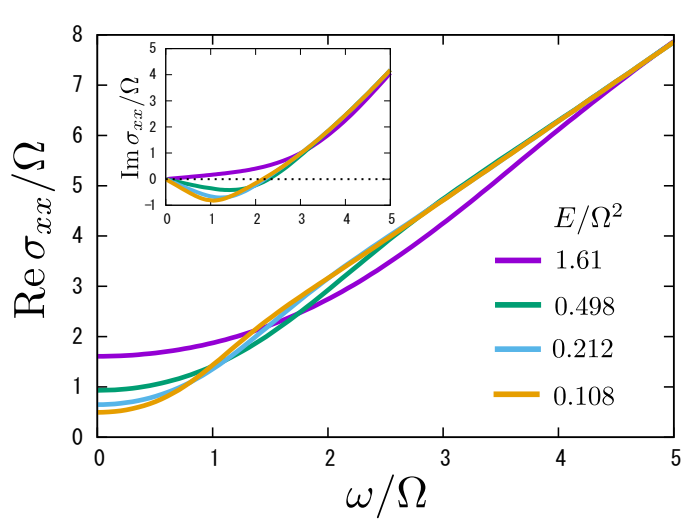

(a) Varying $E$ for fixed $\Omega$

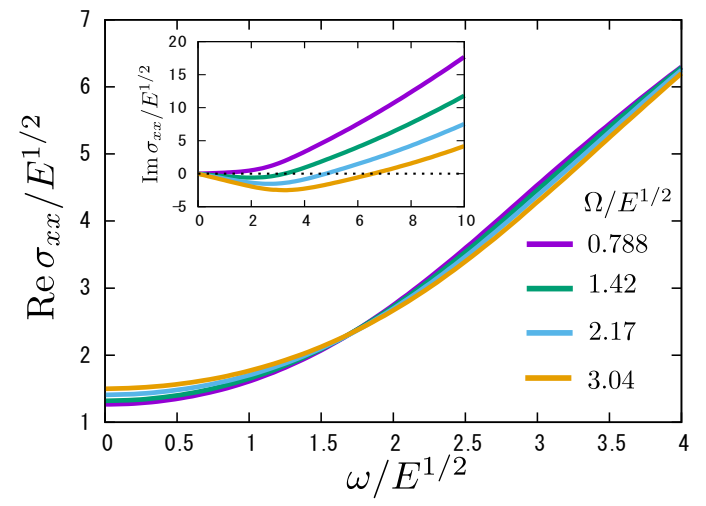

(b) Varying $E$ for fixed $\Omega$

Figure 9. The optical absorption spectrum against the probe AC frequency $\omega$. Curves in the left (right) figure correspond to several $E(\Omega)$ for a fixed $\Omega(E)$.

are amplified and their positions shift to higher frequency as $E(\Omega)$ increases in the left (right) figure. This is consistent with the condensed matter calculation in refs. [27, 55]. The calculation of the optical Hall response done in ref. [27] for a Floquet topological Hall state coupled to a phonon bath shows a striking resemblance with the holographic result figure $8(\mathrm{a})$. They $\left(\operatorname{Re} \sigma_{x y}\right)$ both start from a flat region for small $\omega$ and goes negative at an intermediate frequency, finally approaching zero at $\omega \rightarrow \infty$. In the current system, the gluons not only mediates interaction but also act as a heat bath. This is because we take the large $N_{c}$ limit and the gluons are always in the equilibrium zero-temperature state. In this sense, the NESS obtained here seems to have similar properties as that of ref. [27]. In figure 9, we also show the optical absorption spectrum $\sigma_{x x}$ against $\omega . \operatorname{Re} \sigma_{x x}$ is an increasing function of $E$ and $\Omega$.

The other conductivities $\boldsymbol{\sigma}^{ \pm}$are studied in appendix E.

\section{Conclusion and discussion}

Weyl semimetal can be created from Dirac semimetals by applying rotating electric fields. In this paper, we have considered a similar set up in a strong coupling limit using AdS/CFT correspondence. As a toy model of the strongly coupled field theory, we focused on $\mathcal{N}=2$ $\mathrm{SU}\left(N_{c}\right)$ supersymmetric QCD. Its gravity dual is realized by the probe D7-brane in the $\mathrm{AdS}_{5} \times S^{5}$ spacetime.

We applied the external rotating electric field as a boundary condition of the D-brane and computed its nonlinear response: electric current $j$, Joule heating $q$ and temperature $T_{H}$. We found that they are increasing functions of $\Omega(E)$ for a fixed $E(\Omega)$. For weak electric field $E / \Omega^{2} \ll 1$, the electric current satisfies the Ohm's law $j \propto E$. On the other hand, for low frequency limit $\Omega / E^{1 / 2} \ll 1$, we reproduced the same result as that for DC electric field: $j=E^{3 / 2}$. The non-zero Joule heating implies that there is a steady energy flow from quarks to gluons in the supersymmetric QCD. Since only quarks are electrically charged, they are coherently excited by the electric field. Then, Joule heating will take 
place and the effective temperature will rise. However, the system reaches a steady state since the quarks are coupled with the gluons acting as a heat bath. If we could work with a finite $N_{c}$, it is likely that the gluons will also heat up and eventually the system may drift to an infinite temperature state [23].

We also studied its linear response: we applied probe DC and $\mathrm{AC}$ electric fields in addition to the background rotating electric field. We found Hall currents as their linear response. The Hall effects are expected for Weyl semimetal in free electron picture. Our results suggest that the strong interaction does not wash out the Hall response of Floquet Weyl semimetals. We also find frequency mixed response currents, i.e., a heterodyning effect, characteristic to periodically driven Floquet systems.

In a field theory side, computations of the transport properties of a NESS are usually difficult because we need to know its non-equilibrium distribution of electrons. Once we assume that it is approximated by the equilibrium distribution function, the DC Hall conductivity of a Floquet Weyl semimetal is known to be proportional to the separation of Weyl points in the momentum space $[17,56]$. For the weak coupling limit, as studied in section 2 , the separation of Weyl points is given by $\Delta p \sim E^{2} / \Omega^{3}$ for $E / \Omega^{2} \ll 1$. Thus, the DC Hall conductivity in weak coupling would be given by $\sigma_{x y} / \Omega \sim\left(E / \Omega^{2}\right)^{\alpha}$ with $\alpha=2$ for $E / \Omega^{2} \ll 1$. For strong coupling limit, on the other hand, fitting the plot in figure 7 (a), we obtain $\alpha \simeq 0.7$. This discrepancy suggests that the power $\alpha$ would change at the strongly coupled NESS.

Our analysis opens up a way to analyze nonlinear effects of oscillatory electric field with a circular polarization. In particular, we are interested in how a deconfinement transition can be triggered by tuning the frequency $\Omega$ of the external electric field. It would provide an interesting dynamical phase diagram of QCD-like gauge theories - confinement/deconfinement phase diagram with the axes of the amplitude/frequency of the external electric field. Such kind of study would need holographic approach with an intense and oscillatory electric field, and our method presented here can definitely help. We would like to report on it soon [57].

\section{Acknowledgments}

We would like to thank Leda Bucciantini, Sthitadhi Roy, Sota Kitamura, Kenichi Asano, Hideo Aoki, and Ryo Shimano for valuable discussions. The work of K.H. was supported in part by JSPS KAKENHI Grant Number $15 \mathrm{H} 03658$ and 15K13483. The work of S.K. was supported in part by JSPS KAKENHI Grant Number JP16K17704. The work of K.M. was supported by JSPS KAKENHI Grant Number 15K17658. The work of T.O. was supported by JSPS KAKENHI Grant Number 23740260 and the ImPact project (No. 2015-PM12-05-01) from JST.

\section{A Regular solution near the effective horizon}

We study the regular solution of eq. (3.16) near the effective horizon $\rho=\rho_{c}$. For simplicity, we take the unit of $\Omega=1$ and set $\theta=0$ in this section. We expand $b(\rho)$ near the horizon as

$$
b(\rho)=\rho_{c}^{2}+p\left(\rho-\rho_{c}\right)+\cdots,
$$


where $p$ is a complex constant which will be determined by the regularity. Substituting the above expression into eq. (3.16), we obtain equation for $p$ from the leading term in $\rho-\rho_{c}$ as

$$
\rho_{c} p^{3}-10 \rho_{c} p^{2} p^{*}+\rho_{c} p p^{* 2}-4 p^{2}-8 \rho_{c} p^{*}-4=0 \text {. }
$$

Multiplying (A.2) by $p^{*}$ and taking its imaginary part, we obtain

$$
\left(p-p^{*}\right)\left(p p^{*}-2 \rho_{c} p-2 \rho_{c} p^{*}-1\right)=0 \text {. }
$$

This yields two equations,

$$
p^{*}=p, \quad p^{*}=\frac{2 \rho_{c} p+1}{p-2 \rho_{c}} .
$$

The first equation implies that $p$ is a real value. In this case, the Ricci scalar with respect to the effective metric (3.25) becomes

$$
R(\gamma) \simeq \frac{\rho_{c}^{2}\left(p^{4}+6 p^{2}-16 \rho_{c} p+16 \rho_{c}^{2}+1\right)}{8\left(1+p^{2}\right)\left(p-2 \rho_{c}\right)^{2}\left(\rho-\rho_{c}\right)^{2}} \quad\left(\rho \rightarrow \rho_{c}\right)
$$

The naked singularity appears at $\rho=\rho_{c}$ when $p$ is a real value. So, we consider the second equation in eq. (A.4). Substituting it into eq. (A.2) and dividing it by $p$, we obtain 4 th order equation for $p$ as

$$
\rho_{c}\left(9+32 \rho_{c}^{2}\right)-4\left(1+2 \rho_{c}^{2}\right) p+6 \rho_{c}\left(1+8 \rho_{c}^{2}\right) p^{2}-4\left(1+6 \rho_{c}^{2}\right) p^{3}+\rho_{c} p^{4}=0 .
$$

We have four solutions of this equation as

$$
p=\left\{\begin{array}{l}
\mathcal{R}_{6}-\sqrt{\mathcal{R}_{4} \mathcal{R}_{9}} \pm i \sqrt{2 \mathcal{R}_{4}\left(\sqrt{\mathcal{R}_{4} \mathcal{R}_{9}}-\mathcal{R}_{6}\right)} \\
\mathcal{R}_{6}+\sqrt{\mathcal{R}_{4} \mathcal{R}_{9}} \pm \sqrt{2 \mathcal{R}_{4}\left(\sqrt{\mathcal{R}_{4} \mathcal{R}_{9}}+\mathcal{R}_{6}\right)}
\end{array}\right.
$$

where $\mathcal{R}_{n}$ is defined below eq. (3.18). The latter two solutions do not satisfy the original equation (A.2) and they are fictional solutions. Let us focus on the former two solutions, which are true solutions of (A.2). When we take the positive signature of the former solutions, we find that the Joule heating is positive as in figure 5. On the other hand, if we take the negative signature, the solution is replaced as $b \leftrightarrow b^{*}, E \leftrightarrow-E^{*}$ and $j \leftrightarrow j^{*}$. Then, the Joule heating is replaced as $q \leftrightarrow-q$ and becomes negative. Briefly speaking, this means time reversal solutions. In the view of the effective geometry, there is a energy flux from the white hole horizon. In this paper, we adopt the positive signature of the former solution in eq. (A.7).

\section{B Perturbation equations}

The action for the perturbation of $b$ is obtained by replacing $b(t, \rho)$ with $b(\rho)+\delta b(t, \rho)$ in eq. (3.13) and taking the second order in $\delta b$. Here, $b(\rho)$ is the background solution satisfying eq. (3.16). The equation of motion for $\delta b$ is given by eq. (4.5). Components of 
matrices $\boldsymbol{A}, \boldsymbol{B}$ and $\boldsymbol{C}$ are written as

$$
\begin{aligned}
A_{11}= & 2 \Omega \rho \mathcal{L}_{0}^{-2}\left\{2\left(b_{1} b_{1}^{\prime}+b_{2} b_{2}^{\prime}\right)+\rho\right\}\left(\Omega^{2} b_{1} b_{2}+\rho^{4} b_{1}^{\prime} b_{2}^{\prime}\right), \\
A_{12}= & -2 \Omega \rho \mathcal{L}_{0}^{-2}\left\{2\left(b_{1} b_{1}^{\prime}+b_{2} b_{2}^{\prime}\right)+\rho\right\}\left(-\Omega^{2} b_{2}^{2}+\rho^{4} b_{1}^{\prime 2}+\rho^{4}\right), \\
A_{21}= & -\left(b_{1} \leftrightarrow b_{2} \text { in } A_{12}\right), \\
A_{22}= & -A_{11}, \\
B_{11}= & \mathcal{L}_{0}^{-1}\left[2 \Omega^{2}\left(b_{1} b_{1}^{\prime}-\rho\right)\left(b_{1} b_{1}^{\prime}+b_{2} b_{2}^{\prime}\right)+\Omega^{2}\left(b_{1}^{2}+5 b_{2}^{2}\right)-6 \rho^{4} b_{1}^{\prime 2}-\rho^{4}\right], \\
B_{12}= & 2 \mathcal{L}_{0}^{-1}\left[\Omega^{2} b_{1}^{\prime} b_{2}\left(b_{1} b_{1}^{\prime}+b_{2} b_{2}^{\prime}\right)+\Omega^{2}\left\{\rho\left(b_{1} b_{2}^{\prime}-b_{1}^{\prime} b_{2}\right)-2 b_{1} b_{2}\right\}-3 \rho^{4} b_{1}^{\prime} b_{2}^{\prime}\right], \\
B_{21}= & \left(b_{1} \leftrightarrow b_{2} \text { in } B_{12}\right), \\
B_{22}= & \left(b_{1} \leftrightarrow b_{2} \text { in } B_{11}\right), \\
C_{11}= & -\Omega^{2} \rho \mathcal{L}_{0}^{-2}\left[-2 \Omega^{2} b_{1}^{\prime} b_{2}\left(b_{1} b_{2}^{\prime}-b_{1}^{\prime} b_{2}\right)\left(b_{1} b_{1}^{\prime}+b_{2} b_{2}^{\prime}\right)\right. \\
& +\Omega^{2} \rho\left(b_{1} b_{1}^{\prime}+b_{1} b_{2}^{\prime}-b_{1}^{\prime} b_{2}+b_{2} b_{2}^{\prime}\right)\left(b_{1} b_{1}^{\prime}-b_{1} b_{2}^{\prime}+b_{1}^{\prime} b_{2}+b_{2} b_{2}^{\prime}\right) \\
& +4 \Omega^{2} b_{2}\left(b_{1}^{2} b_{2}^{\prime}-2 b_{1} b_{1}^{\prime} b_{2}-b_{2}^{2} b_{2}^{\prime}\right)+2 \rho^{4} b_{1}^{\prime}\left(2 b_{1} b_{1}^{\prime 2}+3 b_{1} b_{2}^{2}-b_{1}^{\prime} b_{2} b_{2}^{\prime}\right) \\
& \left.+\Omega^{2} \rho\left(b_{1}^{2}-b_{2}^{2}\right)+\rho^{5}\left(b_{1}^{\prime 2}-b_{2}^{\prime 2}\right)+4 \rho^{4}\left(b_{1} b_{1}^{\prime}+b_{2} b_{2}^{\prime}\right)+\rho^{5}\right], \\
C_{12}= & 2 \Omega^{2} \rho \mathcal{L}_{0}^{-2}\left[-\Omega^{2} b_{1} b_{1}^{\prime}\left(b_{1} b_{2}^{\prime}-b_{1}^{\prime} b_{2}\right)\left(b_{1} b_{1}^{\prime}+b_{2} b_{2}^{\prime}\right)\right. \\
& +\Omega^{2} \rho\left(b_{1} b_{2}^{\prime}-b_{1}^{\prime} b_{2}\right)\left(b_{1} b_{1}^{\prime}+b_{2} b_{2}^{\prime}\right)+2 \Omega^{2} b_{1}\left(b_{1}^{2} b_{2}^{\prime}-2 b_{1} b_{1}^{\prime} b_{2}-b_{2}^{2} b_{2}^{\prime}\right) \\
& +b_{1}^{\prime} \rho^{4}\left(b_{1} b_{1}^{\prime} b_{2}^{\prime}-3 b_{1}^{\prime 2} b_{2}-2 b_{2} b_{2}^{\prime 2}\right)-\Omega^{2} \rho b_{1} b_{2} \\
& \left.-2 \rho^{4}\left(b_{1} b_{2}^{\prime}-b_{1}^{\prime} b_{2}\right)-\rho^{5} b_{1}^{\prime} b_{2}^{\prime}\right], \\
C_{21}= & \left(b_{1} \leftrightarrow b_{2} \text { in } C_{12}\right), \\
C_{22}= & \left(b_{1} \leftrightarrow b_{2} \text { in } C_{11}\right), \\
&
\end{aligned}
$$

where $b_{1}=\operatorname{Re} b$ and $b_{2}=\operatorname{Im} b$. We have eliminated $d^{2} b / d \rho^{2}$ using eq. (3.16) in the above expressions.

Now, we study the asymptotic behavior of $\delta \vec{b}$ at the horizon $\rho=\rho_{c}$. The asymptotic form of the background solution $b(\rho)$ is given by eq. (A.1). Substituting eq. (A.1) into $\boldsymbol{A}, \boldsymbol{B}$ and $\boldsymbol{C}$, we obtain

$$
\begin{aligned}
& \boldsymbol{A}_{0} \equiv \boldsymbol{A}\left(\rho=\rho_{c}\right)=\left(\begin{array}{cc}
2 p_{1}\left(2 \rho_{c} p_{1}+1\right) / p_{2} & -2\left(1+p_{1}^{2}\right)\left(2 \rho_{c} p_{1}+1\right) / p_{2}^{2} \\
2\left(2 \rho_{c} p_{1}+1\right) & -2 p_{1}\left(2 \rho_{c} p_{1}+1\right) / p_{2}
\end{array}\right), \\
& \boldsymbol{B}_{0} \equiv \boldsymbol{B}\left(\rho=\rho_{c}\right)=\left(\begin{array}{cc}
-2 p_{1}\left(2 \rho_{c} p_{1}+1\right) / p_{2} & -2\left(3 \rho_{c} p_{1}-1\right) \\
-2\left(2 \rho_{c} p_{1}+1\right) & -2\left(3 \rho_{c} p_{2}^{2}-2 \rho_{c}+p_{1}\right) / p_{2}
\end{array}\right), \\
& \boldsymbol{C}_{0} \equiv \boldsymbol{C}\left(\rho=\rho_{c}\right)=\left(\begin{array}{cc}
-2\left(1+2 \rho_{c} p_{1}+p_{1}^{2}+2 \rho_{c} p_{1}^{3}-p_{2}^{2}+3 \rho_{c} p_{1} p_{2}^{2}\right) / p_{2}^{2} & 0 \\
-2\left(-2 \rho_{c}+2 p_{1}+2 \rho_{c} p_{1}^{2}+3 \rho_{c} p_{2}^{2}\right) / p_{2} & 0
\end{array}\right),
\end{aligned}
$$

where $p_{1}=\operatorname{Re} p$ and $p_{2}=\operatorname{Im} p$. We have not used the explicit expression for $p$ in the above expressions. Substituting the explicit expression of $p$ (the positive signature of the former solution in eq. (A.7)) into the above expressions, we have $\boldsymbol{B}_{0}=-\boldsymbol{A}_{0}$ and $\boldsymbol{C}_{0}=0$. Eventually, near the horizon $\rho=\rho_{c}$, the perturbation equation becomes

$$
\left(\partial_{\tau}^{2}-\partial_{\rho_{*}}^{2}\right) \delta \vec{b}+\boldsymbol{A}_{0}\left(\partial_{\tau}-\partial_{\rho_{*}}\right) \delta \vec{b}=0 .
$$


Therefore, the asymptotic solution is

$$
\delta \vec{b} \simeq \vec{f}\left(t+\rho_{*}\right)+e^{\boldsymbol{A}_{0} \rho_{*}} \vec{g}\left(t-\rho_{*}\right) \quad\left(\rho \rightarrow \rho_{c}\right) .
$$

We will impose $\vec{g}=0$ from the ingoing wave boundary condition.

\section{Relations in conductivity matrices}

We give a proof of the relations in conductivities (4.13). Since the perturbation equations (4.7) are linear, $\beta_{ \pm}^{(0)}$ and $\beta_{ \pm}^{(2)}$ defined in eq. (4.8) are linearly related as

$$
\vec{\beta}_{ \pm}^{(2)}=\boldsymbol{X}_{ \pm} \vec{\beta}_{ \pm}^{(0)}=-\frac{i}{\omega} \boldsymbol{X}_{ \pm} \boldsymbol{M}_{ \pm} \vec{\varepsilon}_{\omega}
$$

where $\boldsymbol{X}_{ \pm}$is a $2 \times 2$ complex matrix. At the last equality, we used eq. (4.9). Substituting the above expressions into eq. (4.11), we obtain

$$
\begin{aligned}
\delta \vec{j} \propto\left[e^{-i \omega t}\left(\boldsymbol{M}_{+} \boldsymbol{X}_{+} \boldsymbol{M}_{+}+\boldsymbol{M}_{-} \boldsymbol{X}_{-} \boldsymbol{M}_{-}\right)\right. & \\
& \left.+e^{-i(\omega+2 \Omega) t} \boldsymbol{M}_{-} \boldsymbol{X}_{+} \boldsymbol{M}_{+}+e^{-i(\omega-2 \Omega) t} \boldsymbol{M}_{+} \boldsymbol{X}_{-} \boldsymbol{M}_{-}\right] \vec{\varepsilon}_{\omega} .
\end{aligned}
$$

Therefore, conductivities are written as

$$
\boldsymbol{\sigma} \propto \boldsymbol{M}_{+} \boldsymbol{X}_{+} \boldsymbol{M}_{+}+\boldsymbol{M}_{-} \boldsymbol{X}_{-} \boldsymbol{M}_{-}, \quad \boldsymbol{\sigma}^{ \pm} \propto \boldsymbol{M}_{\mp} \boldsymbol{X}_{ \pm} \boldsymbol{M}_{ \pm} .
$$

We will denote the components of $\boldsymbol{X}_{ \pm}$as $x_{i j}^{ \pm}(i=1,2)$. By the explicit calculation of matrix multiplications, we obtain

$$
\begin{aligned}
\boldsymbol{\sigma} & \propto\left(x_{11}^{+}+i x_{21}^{+}-i x_{12}^{+}+x_{22}^{+}\right) \boldsymbol{M}_{+}+\left(x_{11}^{-}-i x_{21}^{-}+i x_{12}^{-}+x_{22}^{-}\right) \boldsymbol{M}_{-}, \\
\boldsymbol{\sigma}^{ \pm} & \propto\left(x_{11}^{ \pm} \mp i x_{21}^{ \pm} \mp i x_{12}^{ \pm}-x_{22}^{ \pm}\right)\left(\begin{array}{cc}
1 & \pm i \\
\pm i & -1
\end{array}\right) .
\end{aligned}
$$

From the above expressions, we can find the relations in eq. (4.13).

\section{Numerical method for the time domain approach}

In this section, we explain how to solve the perturbation equation (4.5) in the time domain. We introduce double null coordinates as $u=\left(\tau+\rho_{*}\right) / 2$ and $v=\left(\tau-\rho_{*}\right) / 2$. Then, the perturbation equation becomes

$$
\left[\partial_{u} \partial_{v}+\frac{1}{2}(\boldsymbol{A}+\boldsymbol{B}) \partial_{u}+\frac{1}{2}(\boldsymbol{A}-\boldsymbol{B}) \partial_{v}+\boldsymbol{C}\right] \delta \vec{b}=0
$$

We consider a numerical mesh along $(u, v)$-coordinates as in figure 10. At points shown by white circles $(\circ)$, we give a trivial initial condition: $\delta \vec{b}=0$. At points shown by white squares (๑), we impose the boundary condition in eq. (4.16). To determine the solution inside the numerical domain, we discretize the derivatives at point $\mathrm{C}$ in the figure as

$$
\begin{aligned}
\left.\partial_{u} \partial_{v} \delta \vec{b}\right|_{\mathrm{C}} & =\left(\delta \vec{b}_{\mathrm{N}}-\delta \vec{b}_{\mathrm{E}}-\delta \vec{b}_{\mathrm{W}}+\delta \vec{b}_{\mathrm{S}}\right) / h^{2}, \\
\left.\partial_{u} \delta \vec{b}\right|_{\mathrm{C}} & =\left(\delta \vec{b}_{\mathrm{N}}-\delta \vec{b}_{\mathrm{E}}+\delta \vec{b}_{\mathrm{W}}-\delta \vec{b}_{\mathrm{S}}\right) /(2 h), \\
\left.\partial_{v} \delta \vec{b}\right|_{\mathrm{C}} & =\left(\delta \vec{b}_{\mathrm{N}}+\delta \vec{b}_{\mathrm{E}}-\delta \vec{b}_{\mathrm{W}}-\delta \vec{b}_{\mathrm{S}}\right) /(2 h), \\
\left.\delta \vec{b}\right|_{\mathrm{C}} & =\left(\delta \vec{b}_{\mathrm{E}}+\delta \vec{b}_{\mathrm{W}}\right) / 2
\end{aligned}
$$




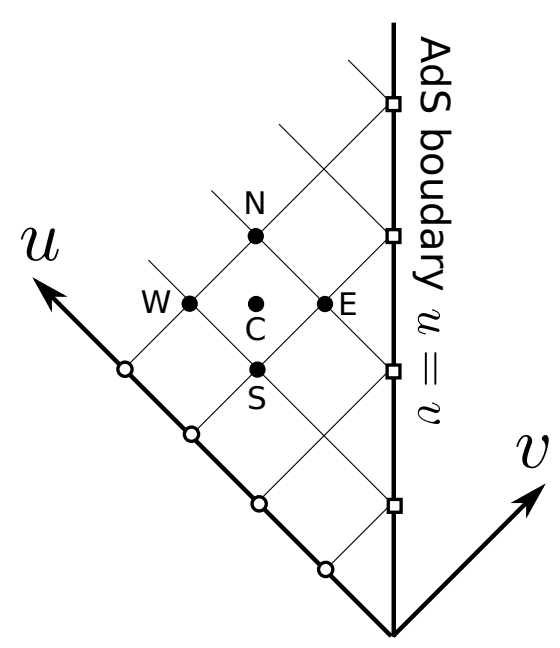

Figure 10. World volume of D7-brane. The mesh for numerical calculation is taken along double null coordinates $u$ and $v$.

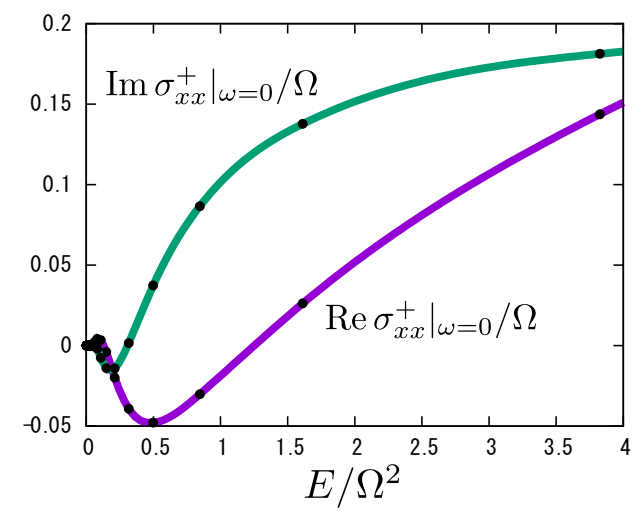

(a) Varying $E$ for fixed $\Omega$

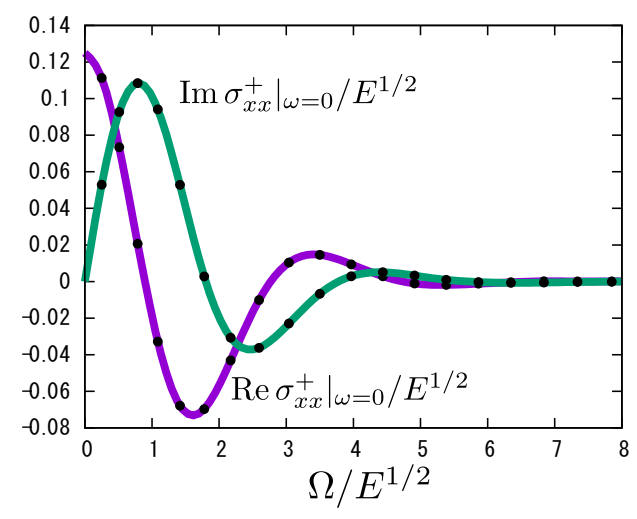

(b) Varying $\Omega$ for fixed $E$

Figure 11. Conductivity $\sigma_{x x}^{+}$against probe DC electric field.

where $h$ is the mesh size and $\delta \vec{b}_{i}(i=\mathrm{N}, \mathrm{E}, \mathrm{W}, \mathrm{S})$ represents numerical value of $\delta \vec{b}$ at point $i$. They have second order accuracy. Substituting the above expressions into eq. (D.1), we can express $\delta \vec{b}_{\mathrm{N}}$ by $\delta \vec{b}_{\mathrm{E}}, \delta \vec{b}_{\mathrm{W}}$ and $\delta \vec{b}_{\mathrm{S}}$. We evaluate $\rho$-dependent matrices $\boldsymbol{A}, \boldsymbol{B}$ and $\boldsymbol{C}$ at the point $\mathrm{C}$. Using the discretized evolution equation sequentially, we can determine the solution in the whole numerical domain.

\section{E Other conductivities}

In section 4.1, we have showed that three modes are induced in the electric current when we apply monochromatic probe AC electric field to the holographic Floquet state. We have defined three kinds of conductivities $\boldsymbol{\sigma}$ and $\boldsymbol{\sigma}^{ \pm}$as in eq. (4.12). In sections 4.2 and 4.3, we have focused only on $\sigma$. Here, we study the other parts of conductivities, $\sigma^{ \pm}$.

Firstly, we consider the probe DC electric field $\omega=0$. In this case, $\left.\sigma^{-}\right|_{\omega=0}$ is given by the complex conjugate of $\left.\boldsymbol{\sigma}^{+}\right|_{\omega=0}$. In figure 11, we show $\left.\sigma_{x x}^{+}\right|_{\omega=0}$ against background 


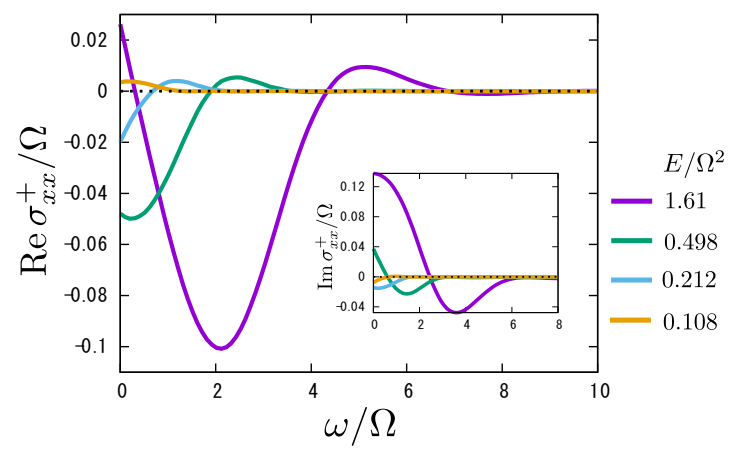

(a) Varying $E$ for fixed $\Omega$

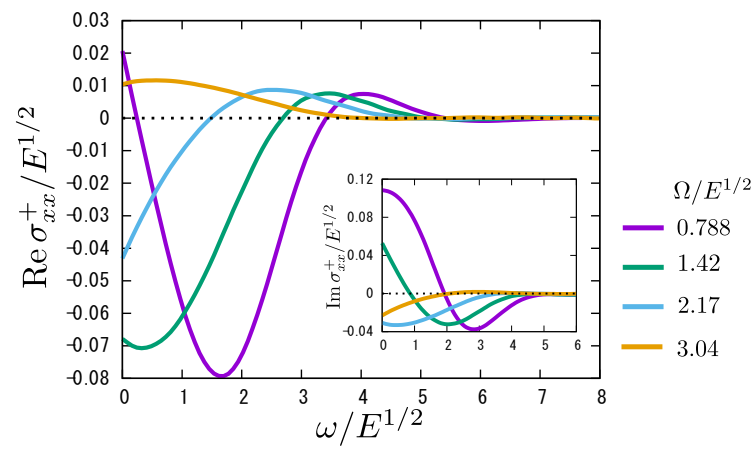

(b) Varying $\Omega$ for fixed $E$

Figure 12. Conductivity $\sigma_{x x}^{+}$against probe $\mathrm{AC}$ electric field.

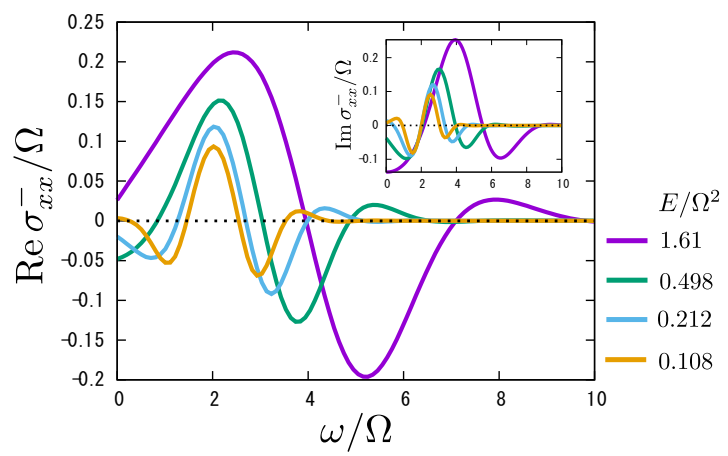

(a) Varying $E$ for fixed $\Omega$

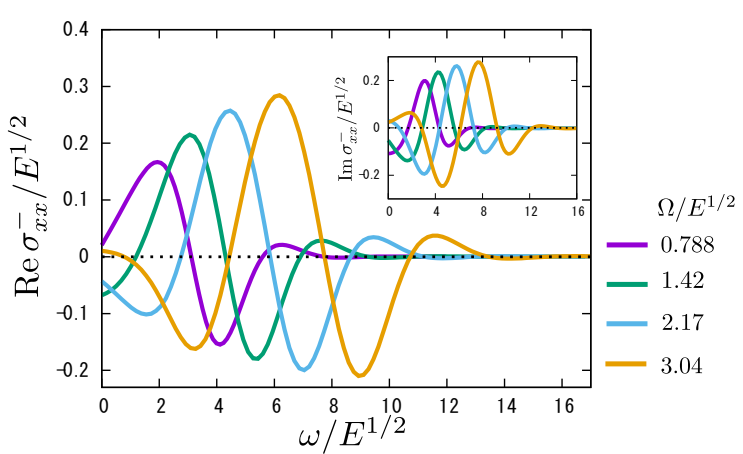

(b) Varying $\Omega$ for fixed $E$

Figure 13. Conductivity $\sigma_{x x}^{-}$against probe $\mathrm{AC}$ electric field.

parameters: (a) $\left.\sigma_{x x}^{+}\right|_{\omega=0} / \Omega$ vs $E / \Omega^{2}$, and (b) $\left.\sigma_{x x}^{+}\right|_{\omega=0} / E^{1 / 2}$ vs $\Omega / E^{1 / 2}$. When we fix the background electric field $E$, the conductivity $\left.\sigma^{+}\right|_{\omega=0}$ oscillates as a function of $\Omega$ for $\Omega \lesssim 4 E^{1 / 2}$ but it is suppressed for $\Omega \gtrsim 4 E^{1 / 2}$. The $\sigma_{+}$expresses the oscillating component of the electric current as one can see in figure 6 . It follows that, for $\Omega \gtrsim 4 E^{1 / 2}$, almost stationary current is induced by the probe DC electric field.

For the probe AC electric field, the conductivities $\sigma_{x x}^{+}$and $\sigma_{x x}^{-}$are shown in figures 12 and 13, respectively. They are all suppressed for large $\omega$. For a fixed $\Omega, \sigma_{x x}^{ \pm}$are amplified as $E$ increases. When we fix $E, \sigma_{x x}^{+}$is getting small as $\Omega$ increases. On the other hand, $\sigma_{x x}^{-}$ becomes large and the position of the peak is shifted to higher frequency as $\Omega$ increases. 
Open Access. This article is distributed under the terms of the Creative Commons Attribution License (CC-BY 4.0), which permits any use, distribution and reproduction in any medium, provided the original author(s) and source are credited.

\section{References}

[1] J.M. Maldacena, The Large-N limit of superconformal field theories and supergravity, Int. J. Theor. Phys. 38 (1999) 1113 [hep-th/9711200] [INSPIRE].

[2] S.S. Gubser, I.R. Klebanov and A.M. Polyakov, Gauge theory correlators from noncritical string theory, Phys. Lett. B 428 (1998) 105 [hep-th/9802109] [INSPIRE].

[3] E. Witten, Anti-de Sitter space and holography, Adv. Theor. Math. Phys. 2 (1998) 253 [hep-th/9802150] [INSPIRE].

[4] S.A. Hartnoll, C.P. Herzog and G.T. Horowitz, Building a Holographic Superconductor, Phys. Rev. Lett. 101 (2008) 031601 [arXiv:0803.3295] [INSPIRE].

[5] M. Cubrović, J. Zaanen and K. Schalm, String Theory, Quantum Phase Transitions and the Emergent Fermi-Liquid, Science 325 (2009) 439 [arXiv:0904.1993] [INSPIRE].

[6] L. Huijse, S. Sachdev and B. Swingle, Hidden Fermi surfaces in compressible states of gauge-gravity duality, Phys. Rev. B 85 (2012) 035121 [arXiv:1112.0573] [INSPIRE].

[7] J. Sonner and A.G. Green, Hawking Radiation and Non-equilibrium Quantum Critical Current Noise, Phys. Rev. Lett. 109 (2012) 091601 [arXiv: 1203.4908] [INSPIRE].

[8] H. Sambe, Steady States and Quasienergies of a Quantum-Mechanical System in an Oscillating Field, Phys. Rev. A 7 (1973) 2203.

[9] N.H. Lindner, G. Refael and V. Galitski, Floquet topological insulator in semiconductor quantum wells, Nat. Phys. 7 (2011) 490.

[10] T. Oka and H. Aoki, Photovoltaic Hall effect in graphene, Phys. Rev. B 79 (2009) 081406R.

[11] T. Kitagawa, M.S. Rudner, E. Berg and E. Demler, Exploring topological phases with quantum walks, Phys. Rev. A 82 (2010) 033429.

[12] T. Kitagawa, T. Oka, A. Brataas, L. Fu and E. Demler, Transport properties of nonequilibrium systems under the application of light: Photoinduced quantum Hall insulators without Landau levels, Phys. Rev. B 84 (2011) 235108 [arXiv:1104.4636].

[13] F.D.M. Haldane, Model for a Quantum Hall Effect without Landau Levels: Condensed-Matter Realization of the 'Parity Anomaly', Phys. Rev. Lett. 61 (1988) 2015 [InSPIRE].

[14] G. Jotzu et al., Experimental realization of the topological Haldane model with ultracold fermions, Nature 515 (2014) 237 [arXiv:1406.7874].

[15] Y.H. Wang, H. Steinberg, P. Jarillo-Herrero and N. Gedik, Observation of Floquet-Bloch states on the surface of a topological insulator, Science $\mathbf{3 4 2}$ (2013) 453 [arXiv:1310.7563] [INSPIRE].

[16] H.B. Nielsen and M. Ninomiya, Adler-Bell-Jackiw anomaly and Weyl fermions in crystal, Phys. Lett. B 130 (1983) 389 [INSPIRE].

[17] R. Wang, B. Wang, R. Shen, L. Sheng and D.Y. Xing Floquet Weyl semimetal induced by topological phase transitions, Europhys. Lett. 105 (2014) 17004 [arXiv:1308.4266]. 
[18] X.-X. Zhang, T.T. Ong and N. Nagaosa, Theory of photoinduced Floquet Weyl semimetal phases, Phys. Rev. B 94 (2016) 235137 [arXiv: 1607.05941] [INSPIRE].

[19] H. Hübener, M.A. Sentef, U. de Giovannini, A.F. Kemper and A. Rubio, Creating stable Floquet-Weyl semimetals by laser-driving of 3D Dirac materials, Nat. Commun. 8 (2017) 13940 [arXiv: 1604.03399].

[20] L. Bucciantini, S. Roy, S. Kitamura, T. Oka and L. Bucciantini, to appear.

[21] Z. Yan and Z. Wang, Tunable Weyl Points in Periodically Driven Nodal Line Semimetals, Phys. Rev. Lett. 117 (2016) 087402 [arXiv:1605.04404].

[22] W. Kohn, Periodic thermodynamics, J. Stat. Phys. 103 (2014) 417.

[23] A. Lazarides, A. Das and R. Moessner, Periodic thermodynamics of isolated systems, Phys. Rev. Lett. 112 (2014) 150401 [arXiv:1401.0164].

[24] A. Lazarides, A. Das and R. Moessner, Equilibrium states of generic quantum systems subject to periodic driving, Phys. Rev. E 90 (2014) 012110.

[25] L. D'Alessio and M. Rigol, Long-time Behavior of Isolated Periodically Driven Interacting Lattice Systems, Phys. Rev. X 4 (2014) 041048.

[26] H. Dehghani, T. Oka and A. Mitra, Dissipative Floquet topological systems, Phys. Rev. B 90 (2014) 195429.

[27] H. Dehghani and A. Mitra, Optical Hall conductivity of a Floquet topological insulator, Phys. Rev. B 92 (2015) 165111 [arXiv:1506.08687].

[28] A. Karch and E. Katz, Adding flavor to AdS/CFT, JHEP 06 (2002) 043 [hep-th/0205236] [INSPIRE].

[29] K. Landsteiner and Y. Liu, The holographic Weyl semi-metal, Phys. Lett. B 753 (2016) 453 [arXiv: 1505.04772] [INSPIRE].

[30] A. Karch and A. O'Bannon, Metallic AdS/CFT, JHEP 09 (2007) 024 [arXiv:0705.3870] [INSPIRE].

[31] T. Albash, V.G. Filev, C.V. Johnson and A. Kundu, Quarks in an external electric field in finite temperature large- $N$ gauge theory, JHEP 08 (2008) 092 [arXiv:0709.1554] [INSPIRE].

[32] J. Erdmenger, R. Meyer and J.P. Shock, AdS/CFT with flavour in electric and magnetic Kalb-Ramond fields, JHEP 12 (2007) 091 [arXiv:0709.1551] [INSPIRE].

[33] K. Hashimoto and T. Oka, Vacuum Instability in Electric Fields via AdS/CFT: Euler-Heisenberg Lagrangian and Planckian Thermalization, JHEP 10 (2013) 116 [arXiv: 1307.7423] [INSPIRE].

[34] K. Hashimoto, T. Oka and A. Sonoda, Magnetic instability in AdS/CFT: Schwinger effect and Euler-Heisenberg Lagrangian of supersymmetric QCD, JHEP 06 (2014) 085 [arXiv: 1403.6336] [INSPIRE].

[35] K. Hashimoto, T. Oka and A. Sonoda, Electromagnetic instability in holographic QCD, JHEP 06 (2015) 001 [arXiv: 1412.4254] [INSPIRE].

[36] K. Hashimoto, S. Kinoshita, K. Murata and T. Oka, Electric Field Quench in AdS/CFT, JHEP 09 (2014) 126 [arXiv:1407.0798] [INSPIRE].

[37] K. Hashimoto, S. Kinoshita, K. Murata and T. Oka, Turbulent meson condensation in quark deconfinement, Phys. Lett. B 746 (2015) 311 [arXiv:1408.6293] [INSPIRE]. 
[38] K. Hashimoto, S. Kinoshita, K. Murata and T. Oka, Meson turbulence at quark deconfinement from AdS/CFT, Nucl. Phys. B 896 (2015) 738 [arXiv:1412.4964] [inSPIRE].

[39] S.A. Hartnoll, C.P. Herzog and G.T. Horowitz, Holographic Superconductors, JHEP 12 (2008) 015 [arXiv:0810.1563] [INSPIRE].

[40] S.A. Hartnoll, C.P. Herzog and G.T. Horowitz, Building a Holographic Superconductor, Phys. Rev. Lett. 101 (2008) 031601 [arXiv:0803.3295] [INSPIRE].

[41] S.A. Hartnoll, Lectures on holographic methods for condensed matter physics, Class. Quant. Grav. 26 (2009) 224002 [arXiv:0903.3246] [INSPIRE].

[42] C.P. Herzog, Lectures on Holographic Superfluidity and Superconductivity, J. Phys. A 42 (2009) 343001 [arXiv:0904.1975] [INSPIRE].

[43] S. Sachdev, Condensed Matter and AdS/CFT, arXiv:1002.2947 [INSPIRE].

[44] W.-J. Li, Y. Tian and H.-b. Zhang, Periodically Driven Holographic Superconductor, JHEP 07 (2013) 030 [arXiv: 1305.1600] [INSPIRE].

[45] M. Natsuume and T. Okamura, The enhanced holographic superconductor: is it possible?, JHEP 08 (2013) 139 [arXiv:1307.6875] [INSPIRE].

[46] T. Oka and L. Bucciantini, Heterodyne Hall effect in a two-dimensional electron gas, Phys. Rev. B 94 (2016) 155133 [arXiv:1607.01041].

[47] J.H. Shirley, Solution of the Schrödinger Equation with a Hamiltonian Periodic in Time, Phys. Rev. 138 (1965) B979.

[48] T. Morimoto and N. Nagaosa, Weyl Mott Insulator, Sci. Rep. 6 (2016) 19853 [arXiv:1508.03203].

[49] K.-Y. Kim, J.P. Shock and J. Tarrio, The open string membrane paradigm with external electromagnetic fields, JHEP 06 (2011) 017 [arXiv: 1103.4581] [INSPIRE].

[50] A. Karch, A. O'Bannon and E. Thompson, The Stress-Energy Tensor of Flavor Fields from AdS/CFT, JHEP 04 (2009) 021 [arXiv:0812.3629] [INSPIRE].

[51] N. Seiberg and E. Witten, String theory and noncommutative geometry, JHEP 09 (1999) 032 [hep-th/9908142] [INSPIRE].

[52] G.W. Gibbons and C.A.R. Herdeiro, Born-Infeld theory and stringy causality, Phys. Rev. D 63 (2001) 064006 [hep-th/0008052] [INSPIRE].

[53] G.W. Gibbons, Pulse propagation in Born-Infeld theory: The World volume equivalence principle and the Hagedorn-like equation of state of the Chaplygin gas, Grav. Cosmol. 8 (2002) 2 [hep-th/0104015] [INSPIRE].

[54] G. Gibbons, K. Hashimoto and P. Yi, Tachyon condensates, Carrollian contraction of Lorentz group and fundamental strings, JHEP 09 (2002) 061 [hep-th/0209034] [INSPIRE].

[55] T. Oka and H. Aoki, All Optical Measurement Proposed for the Photovoltaic Hall Effect, J. Phys. Conf. Ser. 334 (2011) 012060 [arXiv:1007.5399].

[56] A.A. Burkov and L. Balents, Weyl Semimetal in a Topological Insulator Multilayer, Phys. Rev. Lett. 107 (2011) 127205 [arXiv:1105.5138] [INSPIRE].

[57] K. Hashimoto, S. Kinoshita, K. Murata and T. Oka, in preparation. 